\title{
ONE-SIDED INVERTIBILITY OF BINOMIAL FUNCTIONAL OPERATORS WITH A SHIFT IN REARRANGEMENT-INVARIANT SPACES
}

\author{
ALEXEI YU. KARLOVICH* and YURI I. KARLOVICH ${ }^{\dagger}$
}

Let $\Gamma$ be an oriented Jordan smooth curve and $\alpha$ a diffeomorphism of $\Gamma$ onto itself which has an arbitrary nonempty set of periodic points. We prove criteria for one-sided invertibility of the binomial functional operator

$$
A=a I-b W
$$

where $a$ and $b$ are continuous functions, $I$ is the identity operator, $W$ is the shift operator, $W f=f \circ \alpha$, in a reflexive rearrangement-invariant space $X(\Gamma)$ with Boyd indices $\alpha_{X}, \beta_{X}$ and Zippin indices $p_{X}, q_{X}$ satisfying inequalities

$$
0<\alpha_{X}=p_{X} \leq q_{X}=\beta_{X}<1 .
$$

\section{Introduction}

Let $\Gamma$ be an oriented Jordan (i.e., homeomorphic to a circle) smooth curve. Let $\alpha$ be a diffeomorphism of $\Gamma$ onto itself which preserves or changes the orientation on $\Gamma$. We consider the binomial functional operator

$$
A:=a I-b W
$$

acting in a rearrangement-invariant space $X(\Gamma)$, where $a$ and $b$ are continuous functions on $\Gamma, I$ is the identity operator, and $W$ is the shift operator defined by

$$
(W f)(t):=f[\alpha(t)], \quad t \in \Gamma .
$$

An investigation of the two- and one-sided invertibility of functional operators (in particular, (1.1)) in various functional spaces plays an important role in the theory of functional differential operators (see, e.g., [2], [3], [18]), theory of singular integral operators, convolution type operators and pseudodifferential operators with shifts and/or oscillating coefficients (see [4], [16], [20] and the references therein), theory of dynamical systems [9], theory of Banach lattices and Banach $C(K)$-modules [1], etc.

\footnotetext{
*Partially supported by F.C.T. (Portugal) grant PRAXIS XXI/BPD/22006/99.

†Partially supported by CONCACYT (México) grant, Cátedra Patrimonial, No. 990017-EX., nivel II.
} 
Criteria for the two-sided invertibility of the operator (1.1) in Lebesgue spaces $L^{p}(\Gamma), 1<p<\infty$, were obtained by V. G. Kravchenko and the second author [15]. Criteria for one-sided invertiblity of (1.1) in $L^{p}(\Gamma)$ were established by R. Mardiev [23, 24]. These results were extended by $\mathrm{V}$. Aslanov and the second author to the case of reflexive Orlicz spaces $L^{M}(\Gamma)$ and were announced in [6], the full proofs were given in [5, Section 4]. All these results are contained in the survey [14] of the second author. The paper [13] of the first author was devoted to a further generalization and development of ideas and results of [5, 6] to the case of reflexive rearrangement-invariant spaces and shifts having only two fixed points.

In this paper we extend the results of [13] to the case of shifts $\alpha$ preserving or changing the orientation on $\Gamma$ and having an arbitrary nonempty set of periodic points. A nontrivial example of such shift was constructed in [15] (see also [16, p. 74]). We obtain criteria for one-sided invertibility of the functional operator (1.1) in a reflexive rearrangementinvariant space $X(\Gamma)$ of fundamental type with nontrivial Boyd indices. Such spaces are wide generalizations of Lebesgue and Orlicz spaces.

The paper is organized as follows. In Section 2 we formulate necessary properties of rearrangement-invariant spaces and their interpolation characteristics (the Boyd and Zippin indices). We also describe the structure of the set $\Lambda$ of periodic points of the shift $\alpha$ (which have the same multiplicity $m \in \mathbf{N}$ if $\alpha$ preserves the orientation on $\Gamma$ and have multiplicities 1 and 2 otherwise, in the latter case we put $m=2$ ). Difficulties appear in the case of the infinite boundary $\partial \Lambda$ of the set of periodic points. In that case the curve $\Gamma$ to within a finite subset can be represented as a finite union of pairwise disjoint open arcs of three types: on the arcs of first type the shift $\alpha$ is Carleman (that is, $\alpha_{m}(t) \equiv t$ ), on the arcs of second type the shift $\alpha_{m}$ has only two fixed points (the endpoints of the arc), and the closure of the union of the arcs of third type contains the set $(\partial \Lambda)^{\prime}$ of all limit points of the boundary $\partial \Lambda$ of $\Lambda$. Moreover, we can choose the arcs of third type so small as we want. All these arcs are invariant with respect to the shift $\alpha_{m}$.

In Section 3 we prove sufficient conditions for the two-sided invertibility of the functional operator (1.1) in $X(\Gamma)$. These results are based on an estimate from above for the spectral radius of the weighted shift operator $g W(g \in C(\Gamma))$ in rearrangement-invariant spaces, which is obtained with the help of interpolation from known results for Lebesgue spaces.

Section 4 is devoted to criteria for one-sided invertibility of the functional operator (1.1) in $X(\Gamma)$ in the case of an arbitrary nonempty set of fixed points of $\alpha$. In their proofs we essentially use a decomposition of $\Gamma$ into a union of arcs of three types. Further, for the functional operator (1.1) we define five sets $\Gamma_{j}, j \in\{1,2, \ldots, 5\}$, and in terms of these sets we prove a criterion for one-sided invertibility of $A$. Roughly speaking, $\Gamma_{1}$ is a Carleman part of $\Gamma$, the sets $\Gamma_{2}$ and $\Gamma_{3}$ control the two-sided invertibility of $A$ if, respectively, the coefficient $a$ or $b$ dominates, the set $\Gamma_{4}$ controls the right invertibility of $A$, and the set $\Gamma_{5}$ controls the left invertibility of $A$. Stress also that according to Corollary 4.3, if $\alpha$ has a finite set of fixed points on $\Gamma$, then for considered rearrangement-invariant spaces (with the non-coinciding Boyd indices) in contrast to Lebesgue spaces, the intersection of the left and right spectra of the shift operator $W$ consists of a finite union of annuli depending on the 
values of the derivative $\alpha^{\prime}$ at the fixed points of $\alpha$ and the both Boyd indices. In the case of Lebesgue spaces these annuli degenerate into circles. This shows a new quality arising for functional operators in rearrangement-invariant spaces in view of the non-coincidence, in general, of the Boyd indices.

In Section 5 we extend the results of Section 4 to the case of periodic points of arbitrary multiplicity $m$. The proof is based on the equivalence of the right invertibility of $A$ and the conjunction of the right invertibility of an operator of the form (1.1), with the shift $\alpha_{m}$ having only fixed points, and some additional conditions. Using that equivalence, we reduce the general case to the case of fixed points. The left invertiblity is studied by passing to adjoint operators and making use of the reflexivity of the space $X(\Gamma)$. At the end of this section we calculate the spectrum of the weighted shift operator $g W$ with the continuous coefficient $g$.

\section{Spaces and shifts}

\subsection{Rearrangement-invariant spaces}

For a general discussion of rearrangement-invariant spaces, see [7, 17, 19]. In this subsection we collect necessary facts in the abstract setting of (finite) measure spaces.

Let $(\mathcal{R}, \mu)$ be a nonatomic finite measure space. Denote by $\mathcal{M}=\mathcal{M}(\mathcal{R}, \mu)$ the set of all $\mu$-measurable complex-valued functions on $\mathcal{R}$, and let $\mathcal{M}^{+}$be the subset of functions from $\mathcal{M}$ whose values lie in $[0, \infty]$. The characteristic function of a $\mu$-measurable set $E \subset \mathcal{R}$ will be denoted by $\chi_{E}$. A mapping $\rho: \mathcal{M}^{+} \rightarrow[0, \infty]$ is called a function norm if for all functions $f, g, f_{n} \in \mathcal{M}^{+}(n \in \mathbf{N})$, for all constants $a \geq 0$ and for all $\mu$-measurable subsets $E$ of $\mathcal{R}$, the following properties hold:

(i) $\quad \rho(f)=0 \Leftrightarrow f=0 \mu$-a.e., $\rho(a f)=a \rho(f), \rho(f+g) \leq \rho(f)+\rho(g)$,

(ii) $\quad 0 \leq g \leq f \mu$-a.e. $\Rightarrow \rho(g) \leq \rho(f) \quad$ (the lattice property),

(iii) $\quad 0 \leq f_{n} \uparrow f \mu$-a.e. $\Rightarrow \rho\left(f_{n}\right) \uparrow \rho(f) \quad$ (the Fatou property),

(iv) $\quad \rho\left(\chi_{E}\right)<\infty, \quad \int_{E} f d \mu \leq C_{E} \rho(f)$

with $C_{E} \in(0, \infty)$ depending on $E$ and $\rho$ but independent of $f$. The collection $X=X(\rho)$ of all functions $f \in \mathcal{M}$ for which $\rho(|f|)<\infty$ is called a Banach function space. For each $f \in X$, the norm of $f$ is defined by

$$
\|f\|_{X}:=\rho(|f|)
$$

If $\rho$ is a function norm, its associate norm $\rho^{\prime}$ is defined on $\mathcal{M}^{+}$by

$$
\rho^{\prime}(g):=\sup \left\{\int_{\mathcal{R}} f g d \mu: f \in \mathcal{M}^{+}, \rho(f) \leq 1\right\}, \quad g \in \mathcal{M}^{+} .
$$

The Banach function space $X\left(\rho^{\prime}\right)$ determined by the function norm $\rho^{\prime}$ is called the associate space of $X=X(\rho)$ and is denoted by $X^{\prime}$. The associate space $X^{\prime}$ is a subspace of the dual space $X^{*}$. 
In the following we will consider only separable measure spaces. Note that the Lebesgue measure is separable (for the definition and the proof of this fact, see, e.g., [12, Ch. 1, Subsection 6.10]).

Lemma 2.1. Let $\mu$ be a separable measure.

(a) A Banach function space $X$ is separable if and only if its associate space $X^{\prime}$ is canonically isometrically isomorphic to the dual space $X^{*}$ of $X$.

(b) A Banach function space $X$ is reflexive if and only if both $X$ and its associate space $X^{\prime}$ are separable.

This lemma follows from Corollaries 4.3, 4.4 and 5.6 [7, Ch. 1].

Let $\mathcal{M}_{0}$ and $\mathcal{M}_{0}^{+}$be the classes of $\mu$-a.e. finite functions in $\mathcal{M}$ and $\mathcal{M}^{+}$, respectively. Two functions $f, g \in \mathcal{M}_{0}$ are said to be equimeasurable if

$$
\mu\{x \in \mathcal{R}:|f(x)|>\lambda\}=\mu\{x \in \mathcal{R}:|g(x)|>\lambda\} \text { for all } \lambda \geq 0 .
$$

A function norm $\rho: \mathcal{M}^{+} \rightarrow[0, \infty]$ is said to be rearrangement-invariant, if $\rho(f)=\rho(g)$ for every pair of equimeasurable functions $f, g \in \mathcal{M}_{0}^{+}$. In that case, the Banach function space $X=X(\rho)$ generated by $\rho$ is said to be the rearrangement-invariant space (briefly r.-i. space). Lebesgue, Orlicz, Lorentz spaces are important classical examples of r.-i. spaces.

\subsection{Boyd and Zippin indices}

A measurable function $\varrho:(0, \infty) \rightarrow(0, \infty)$ is said to be submultiplicative if

$$
\varrho\left(x_{1} x_{2}\right) \leq \varrho\left(x_{1}\right) \varrho\left(x_{2}\right) \text { for all } x_{1}, x_{2} \in(0, \infty) .
$$

The behavior of the measurable submultiplicative function $\varrho$ in neighborhoods of zero and infinity is described by the quantities (see [17, Ch. 2, Theorem 1.3])

$$
\alpha(\varrho):=\sup _{x \in(0,1)} \frac{\log \varrho(x)}{\log x}=\lim _{x \rightarrow 0} \frac{\log \varrho(x)}{\log x}, \quad \beta(\varrho):=\inf _{x \in(1, \infty)} \frac{\log \varrho(x)}{\log x}=\lim _{x \rightarrow \infty} \frac{\log \varrho(x)}{\log x} .
$$

One can prove that $\alpha(\varrho) \leq \beta(\varrho)$ and these numbers are finite. The numbers $\alpha(\varrho)$ and $\beta(\varrho)$ are called the lower and upper indices of the measurable submultiplicative function $\varrho$.

The idea of using indices of some submultiplicative functions for the description of properties of Orlicz spaces goes back to W. Matuszewska and W. Orlicz, 1960. MatuszewskaOrlicz indices were generalized by D. W. Boyd and M. Zippin to the case of rearrangementinvariant spaces (for the history and precise references, see [21]).

By the Luxemburg representation theorem [7, Ch. 2, Theorem 4.10], there is the unique rearrangement-invariant function norm $\bar{\rho}$ over $[0, \mu(\mathcal{R})]$ with Lebesgue measure $m$ such that

$$
\rho(f)=\bar{\rho}\left(f^{*}\right) \quad \text { for all } \quad f \in \mathcal{M}_{0}^{+}
$$

where $f^{*}$ is the non-increasing rearrangement of $f$ (see, e.g., [7, p. 39]). The r.-i. space over $([0, \mu(\mathcal{R})], m)$ generated by $\bar{\rho}$ is called the Luxemburg representation of $X$ and is denoted by $\bar{X}$. For each $x>0$, let $E_{x}$ denote the dilation operator defined on $\mathcal{M}_{0}([0, \mu(\mathcal{R})], m)$ by

$$
\left(E_{x} f\right)(t):=\left\{\begin{array}{ll}
f(x t), & x t \in[0, \mu(\mathcal{R})] \\
0, & x t \notin[0, \mu(\mathcal{R})]
\end{array}, \quad t \in[0, \mu(\mathcal{R})] .\right.
$$


Together with $E_{x}$ consider the family of operators

$$
E_{x}^{(\lambda)}:=\Pi_{\lambda} E_{x} \Pi_{\lambda}, \quad \lambda \in(0, \mu(\mathcal{R})]
$$

where $\Pi_{\lambda}:=\chi_{\lambda} I$ and $\chi_{\lambda}$ is the characteristic function of the segment $[0, \lambda]$. Consider the function

$$
h_{X}(x, \lambda):=\left\|E_{1 / x}^{(\lambda)}\right\|_{\mathcal{B}(\bar{X})}, \quad x \in(0, \infty), \quad \lambda \in(0, \mu(\mathcal{R})],
$$

where $\mathcal{B}(\bar{X})$ is the Banach algebra of the bounded linear operators on $\bar{X}$.

Lemma 2.2. (a) For every $x \in(0, \infty)$, we have $h_{X}(x, \mu(\mathcal{R})) \leq \max \{1, x\}$;

(b) for $x \in(0, \infty)$, the function $h_{X}(x, \lambda)$ is non-decreasing in $\lambda \in(0, \mu(\mathcal{R})]$;

(c) for $\lambda \in(0, \mu(\mathcal{R})]$, the function $h_{X}(x, \lambda)$ is non-decreasing and submultiplicative in $x \in(0, \infty)$;

(d) if $X^{\prime}$ denotes the associate space of $X$, then

$$
h_{X}(x, \lambda)=x h_{X^{\prime}}\left(\frac{1}{x}, \lambda\right), \quad x \in(0, \infty), \quad \lambda \in(0, \mu(\mathcal{R})]
$$

(e) if $x \in(0,1]$ and $\lambda \in(0, \mu(\mathcal{R}) / 2]$, then $h_{X}(x, 2 \lambda) \leq 2 h_{X}(x, \lambda)$;

(f) if $0<\lambda \leq \nu \leq \mu(\mathcal{R})$, then there is a constant $C_{\lambda, \nu}>0$ such that

$$
h_{X}(x, \lambda) \leq h_{X}(x, \nu) \leq C_{\lambda, \nu} h_{X}(x, \lambda), \quad x \in(0, \infty) .
$$

Proof. The statement (a) is well-known (see, e.g., [7, p. 165]).

(b) If $\lambda_{1}<\lambda_{2}$, then $\Pi_{\lambda_{1}}=\Pi_{\lambda_{1}} \Pi_{\lambda_{2}}=\Pi_{\lambda_{2}} \Pi_{\lambda_{1}}$. Hence,

$$
E_{1 / x}^{\left(\lambda_{1}\right)}=\Pi_{\lambda_{1}} E_{1 / x} \Pi_{\lambda_{1}}=\Pi_{\lambda_{1}} \Pi_{\lambda_{2}} E_{1 / x} \Pi_{\lambda_{2}} \Pi_{\lambda_{1}}=\Pi_{\lambda_{1}} E_{1 / x}^{\left(\lambda_{2}\right)} \Pi_{\lambda_{1}} .
$$

Since $\left\|\Pi_{\lambda_{1}}\right\|_{\mathcal{B}(\bar{X})} \leq 1$, we infer from (2.4) that

$$
h_{X}\left(x, \lambda_{1}\right)=\left\|E_{1 / x}^{\left(\lambda_{1}\right)}\right\|_{\mathcal{B}(\bar{X})} \leq\left\|E_{1 / x}^{\left(\lambda_{2}\right)}\right\|_{\mathcal{B}(\bar{X})}=h_{X}\left(x, \lambda_{2}\right) .
$$

(c) and (d). For every $x \in(0, \infty)$ and every $\lambda \in(0, \mu(\mathcal{R})]$, we have

$$
\left(E_{x}^{(\lambda)} f\right)^{*}(t) \leq E_{x}^{(\lambda)} f^{*}(t), \quad t \in(0, \mu(\mathcal{R})] .
$$

Using this inequality, one can obtain by analogy with the remark after [0], Ch. 3, Corollary 6.11] that the function $h_{X}(x, \lambda)$ is non-decreasing and submultiplicative in $x \in(0, \infty)$. Moreover, (2.3) holds.

(e) From (2.4), (2.5) and the monotonicity of $f^{*}$ we see that for $t \in(0, \mu(\mathcal{R})]$,

$$
\begin{aligned}
& \left(E_{1 / x}^{(2 \lambda)} f\right)^{*}(t) \leq E_{1 / x}^{(2 \lambda)} f^{*}(t)=E_{1 / x}^{(2 \lambda)}\left(\chi_{\lambda} f^{*}\right)(t)+E_{1 / x}^{(2 \lambda)}\left(\chi_{[\lambda, 2 \lambda]} f^{*}\right)(t) \\
& =\chi_{\lambda}(t) E_{1 / x}^{(2 \lambda)}\left(\chi_{\lambda} f^{*}\right)(t)+E_{1 / x}^{(2 \lambda)}\left(\chi_{[\lambda, 2 \lambda]} f^{*}\right)(t)=E_{1 / x}^{(\lambda)} f^{*}(t)+E_{1 / x}^{(2 \lambda)}\left(\chi_{[\lambda, 2 \lambda]} f^{*}\right)(t) \\
& \left(E_{1 / x}^{(2 \lambda)}\left(\chi_{[\lambda, 2 \lambda]} f^{*}\right)\right)^{*}(t) \leq E_{1 / x}^{(2 \lambda)}\left(\chi_{[\lambda, 2 \lambda]} f^{*}\right)^{*}(t)=E_{1 / x}^{(2 \lambda)}\left(\chi_{\lambda}(t) f^{*}(t+\lambda)\right) \\
& \leq E_{1 / x}^{(2 \lambda)}\left(\chi_{\lambda} f^{*}\right)(t)=\chi_{\lambda}(t) E_{1 / x}^{(2 \lambda)}\left(\chi_{\lambda} f^{*}\right)(t)=E_{1 / x}^{(\lambda)} f^{*}(t)
\end{aligned}
$$


Inequalities (2.6), (2.7) and properties of the r.-i. invariant function norm $\bar{\rho}$ give

$$
\bar{\rho}\left(\left|E_{1 / x}^{(2 \lambda)} f\right|\right)=\bar{\rho}\left(\left(E_{1 / x}^{(2 \lambda)} f\right)^{*}\right) \leq 2 \bar{\rho}\left(E_{1 / x}^{(\lambda)} f^{*}\right)
$$

Obviously, $\left\{f^{*}: f \in \bar{X}, \bar{\rho}(|f|) \leq 1\right\} \subset\{|f|: f \in \bar{X}, \bar{\rho}(|f|) \leq 1\}$. Hence, from (2.8) we get

$$
\begin{aligned}
& h_{X}(x, 2 \lambda)=\left\|E_{1 / x}^{(2 \lambda)}\right\|_{\mathcal{B}(\bar{X})}=\sup _{f \in \bar{X}, \bar{\rho}(|f|) \leq 1} \bar{\rho}\left(\left|E_{1 / x}^{(2 \lambda)} f\right|\right) \leq 2 \sup _{f \in \bar{X}, \bar{\rho}(|f|) \leq 1} \bar{\rho}\left(E_{1 / x}^{(\lambda)} f^{*}\right) \\
& \leq 2 \sup _{f \in \bar{X}, \bar{\rho}(|f|) \leq 1} \bar{\rho}\left(E_{1 / x}^{(\lambda)}|f|\right)=2 \sup _{f \in \bar{X}, \bar{\rho}(|f|) \leq 1} \bar{\rho}\left(\left|E_{1 / x}^{(\lambda)} f\right|\right)=2\left\|E_{1 / x}^{(\lambda)}\right\|_{\mathcal{B}(\bar{X})}=2 h_{X}(x, \lambda) .
\end{aligned}
$$

(f) Choose $n \in \mathbf{N}$ such that $\nu / 2^{n} \leq \lambda<\nu / 2^{n-1}$. Then from (b) and (e) we get

$$
h_{X}(x, \lambda) \leq h_{X}(x, \nu) \leq 2^{n} h_{X}\left(x, \nu / 2^{n}\right) \leq 2^{n} h_{X}(x, \lambda), \quad x \in(0,1] .
$$

If $x \in(1, \infty)$, then from (2.3) and (2.9) we get the same inequality (2.9) for $x \in(1, \infty)$.

The indices of the non-decreasing (and hence, measurable) and submultiplicative function $h_{X}(\cdot, \mu(\mathcal{R}))$ are called the Boyd indices of the r.-i. space $X$ \& and denoted by

$$
\alpha_{X}:=\alpha\left(h_{X}(\cdot, \mu(\mathcal{R}))\right), \quad \beta_{X}:=\beta\left(h_{X}(\cdot, \mu(\mathcal{R}))\right) .
$$

From Lemma 2.2(a), (d) and equalities (2.1) it follows that

$$
\begin{gathered}
0 \leq \alpha_{X} \leq \beta_{X} \leq 1 \\
\alpha_{X}+\beta_{X^{\prime}}=\alpha_{X^{\prime}}+\beta_{X}=1 .
\end{gathered}
$$

For each $t \in(0, \mu(\mathcal{R})]$, let $\Omega$ be a $\mu$-measurable subset of $\mathcal{R}$ with $\mu(\Omega)=t$, and let $\varphi_{X}(t):=\left\|\chi_{\Omega}\right\|_{X}$. The function $\varphi_{X}$ so defined is called the fundamental function of the r.-i. space $X$. Put

$$
M_{X}(x):=\limsup _{t \rightarrow 0} \frac{\varphi_{X}(x t)}{\varphi_{X}(t)}, \quad x \in(0, \infty) .
$$

The function $M_{X}$ is non-decreasing (and hence, measurable) and submultiplicative (see 21, Section 4]). The indices of this function are called the Zippin (or fundamental) indices of the r.-i. space $X$ 25] and denoted by $p_{X}:=\alpha\left(M_{X}\right), q_{X}:=\beta\left(M_{X}\right)$. Generally, it can be proved (see, e.g., [21, Section 4]) that

$$
\alpha_{X} \leq p_{X} \leq q_{X} \leq \beta_{X}
$$

Lemma 2.3. Let $(\mathcal{R}, \mu)$ be a finite measure space and $\Omega$ be a $\mu$-measurable subset of $\mathcal{R}$ with $\lambda:=\mu(\Omega)>0$. Let $X$ be an r.-i. space over $(\mathcal{R}, \mu)$ generated by an r. $i$. function norm $\rho$. Then its subspace $P_{\Omega} X$, where $P_{\Omega}:=\chi_{\Omega} I$, is an $r .-i$. space over $(\Omega, \mu)$ generated by the same r.-i. function norm $\rho$, but defined on functions with support in $\Omega$. Moreover, $X$ and $P_{\Omega} X$ have the same Boyd and Zippin indices. 
Proof. It is easy to check that an r.-i. function norm $\rho$ defined on $\mathcal{M}^{+}(\mathcal{R}, \mu)$ is a Banach function norm on $\mathcal{M}^{+}(\Omega, \mu)$ as well. Moreover, $\rho$ is an r.-i. function norm on $\mathcal{M}^{+}(\Omega, \mu)$. Hence, $P_{\Omega} X$ is an r.-i. space.

It is easy also to see that $\Pi_{\lambda} \bar{X}$ is the Luxemburg representation for the subspace $P_{\Omega} X$, and

$$
\left\|E_{1 / x}^{(\lambda)}\right\|_{\mathcal{B}(\bar{X})}=\left\|E_{1 / x}\right\|_{\mathcal{B}\left(\Pi_{\lambda} \bar{X}\right)},
$$

where the operator $E_{1 / x}$ from the right is defined by (2.2) on functions in $\mathcal{M}_{0}^{+}([0, \lambda], m)$. From (2.1), (2.11) and Lemma 2.2(f) it follows that the Boyd indices of $X$ and $P_{\Omega} X$ coincide.

On the other hand, $\varphi_{X}(t)=\varphi_{P_{\Omega} X}(t)$ for every $t \in(0, \lambda]$. Hence, $M_{X}(x)=M_{P_{\Omega} X}(x)$ for every $x \in(0, \infty)$. Consequently, the Zippin indices of $X$ and $P_{\Omega} X$ coincide too.

An r.-i. space $X$ is said to be of fundamental type if its Boyd and Zippin indices coincide:

$$
\alpha_{X}=p_{X}, \quad \beta_{X}=q_{X} .
$$

Lebesgue, Orlicz, and Lorentz spaces are examples of spaces of fundamental type [10]. For the Lebesgue spaces $L^{p}, 1 \leq p \leq \infty$, all indices are equal to $1 / p$. But there are r.-i. spaces for which the Boyd and Zippin indices do not coincide, that is, there exist r.-i. spaces of non-fundamental type (see [21] and the references given there). We will say that the Boyd indices are nontrivial if

$$
0<\alpha_{X} \leq \beta_{X}<1
$$

In the case of Orlicz spaces these inequalities are equivalent to the reflexivity of the space (see, e.g., [21]). Examples of Young functions which generate reflexive Orlicz spaces whose Boyd indices do not coincide are given in [6] and [22, p. 93].

Boyd indices are interpolation characteristics of r.-i. spaces.

Theorem 2.4 (see [8] and [13, Theorem 2.2]). Let $X$ be an r.-i. space with nontrivial Boyd indices. If there are numbers $p_{0}, p_{1} \in(1, \infty)$ such that

$$
1 / p_{1}<\alpha_{X} \leq \beta_{X}<1 / p_{0},
$$

and if an operator $T$ is bounded in the Lebesgue spaces $L^{p_{0}}$ and $L^{p_{1}}$, then $T$ is bounded in $X$ and

$$
r(T ; X) \leq \max \left\{r\left(T ; L^{1 / \alpha_{X}}\right), r\left(T ; L^{1 / \beta_{X}}\right)\right\},
$$

where $r(T ; X)$ is the spectral radius of $T$ in the r. $-i$. space $X$, and $r\left(T ; L^{1 / \alpha_{X}}\right)$ and $r\left(T ; L^{1 / \beta_{X}}\right)$ are the spectral radii of $T$ in the Lebesgue spaces $L^{1 / \alpha_{X}}$ and $L^{1 / \beta_{X}}$, respectively.

Note that the estimate (2.12) is sharp, that is, there is an operator $T$, for which we have the equality in (2.12) (see Subsection 5.5).

\subsection{Structure of the set of periodic points}

In the following we will consider Jordan curves or arcs of two types. We say that an arc $\gamma$ is closed (open) if it is homeomorphic to the segment $[0,1]$ (to the interval $(0,1)$ ). Let $\Gamma$ be 
an oriented Jordan smooth curve. A homeomorphism $\alpha: \Gamma \rightarrow \Gamma$ is called a shift function (shift). Put $\alpha_{0}(t)=t$ and $\alpha_{n}(t)=\alpha\left[\alpha_{n-1}(t)\right]$ for $n \in \mathbf{Z}$ and $t \in \Gamma$.

A point $\tau \in \Gamma$ is called a periodic point of the multiplicity $m \geq 1$ for the shift $\alpha$, if $\alpha_{m}(\tau)=\tau$ and (in the case $\left.m>1\right) \alpha_{j}(\tau) \neq \tau$ for every $j=1,2, \ldots, m-1$. A periodic point of the multiplicity one is called a fixed point. Let $\Lambda$ be the set of all periodic points. A classification of shifts with respect to the set $\Lambda$ is given in [16, Ch. 1, Section 3]. In the following we assume that the set $\Lambda$ is nonempty. It is known that if $\alpha$ preserves the orientation on $\Gamma$ then all periodic points of $\alpha$ have the same multiplicity [16, Theorem 1.3.1]. If $\alpha$ changes the orientation on $\Gamma$ then $\alpha$ has two fixed points on $\Gamma$, and all other periodic points of $\alpha$ (if they exist) have the multiplicity two [16, Theorem 1.3.2]. Clearly, the set $\Lambda$ is closed. Let $m$ be the multiplicity of periodic points of the shift $\alpha$ if $\alpha$ preserves the orientation on $\Gamma$, and put $m:=2$ otherwise. Then $\Lambda$ is the set of all fixed points of the shift $\alpha_{m}$. Put

$$
\Phi:=\overline{\left\{t \in \Gamma: \alpha_{m}(t) \neq t\right\}}
$$

and decompose the contour $\Gamma$ into disjoint sets

$$
\Gamma=(\Gamma \backslash \Phi) \cup \Phi=(\Gamma \backslash \Phi) \cup(\Phi \backslash \Lambda) \cup Y
$$

where the set $Y:=\partial \Lambda=\Lambda \cap \Phi$ is the boundary of $\Lambda$. Clearly,

$$
\Phi \backslash \Lambda=\Gamma \backslash \Lambda, \quad \text { and } \quad(\Gamma \backslash \Phi) \cup Y=\Lambda
$$

The open set $\Phi \backslash \Lambda$ can be represented as a countable (or finite) union of connected components (open arcs), every of which is invariant with respect to the shift $\alpha_{m}$ ( $\alpha_{m}$-invariant) and does not contain its fixed points. The open set $\Gamma \backslash \Phi$ also can be represented as a countable (or finite) union of $\alpha_{m}$-invariant open arcs but consisting of inner points of $\Lambda$.

Let $Y^{\prime}$ denote the set of all limit points of the boundary $Y$ of $\Lambda$.

Lemma 2.5. (a) If the set $Y$ is finite, then there is a finite decomposition

$$
\Gamma=\left(\bigcup_{i} \overline{\omega_{i}}\right) \cup\left(\bigcup_{j} \overline{\gamma_{j}}\right)
$$

where $\omega_{i} \subset \Gamma \backslash \Phi, \gamma_{j} \subset \Phi \backslash \Lambda$ are pairwise disjoint, $\alpha_{m}$-invariant open arcs with endpoints in $Y$.

(b) Let $f: \Gamma \rightarrow \mathbf{R}$ be a continuous function. If the set $Y$ is infinite and $f(\tau)>0$ for all $\tau \in Y^{\prime}$, then there is a finite decomposition

$$
\Gamma=\left(\bigcup_{i} \overline{\omega_{i}}\right) \cup\left(\bigcup_{j} \overline{\gamma_{j}}\right) \cup\left(\bigcup_{k} \overline{v_{k}}\right),
$$

where $\omega_{i} \subset \Gamma \backslash \Phi, \gamma_{j} \subset \Phi \backslash \Lambda, v_{k} \subset \Gamma$ are pairwise disjoint, $\alpha_{m}$-invariant open arcs with endpoints in $Y, \overline{v_{k}} \cap Y^{\prime} \neq \emptyset$, and $f(t)>0$ for all $t \in \overline{v_{k}}$ and all arcs $v_{k}$. 
Proof. If the set $Y$ is finite, then the set $\Gamma \backslash Y=(\Gamma \backslash \Phi) \cup(\Phi \backslash \Lambda)$ consists of a finite set of pairwise disjoint open arcs with endpoints in $Y$. Since the set $Y$ consists of fixed points of $\alpha_{m}$, these arcs are $\alpha_{m}$-invariant. Denoting these arcs lying in $\Gamma \backslash \Phi$ and $\Phi \backslash \Lambda$ by $\omega_{i}$ and $\gamma_{j}$, respectively, we get

$$
\Gamma \backslash \Phi=\bigcup_{i} \omega_{i}, \quad \Phi \backslash \Lambda=\bigcup_{j} \gamma_{j},
$$

which proves part (a).

(b) If the set $Y$ is infinite, then the set $Y^{\prime}$ of its limit points is nonempty, and vice versa. Moreover, since $Y^{\prime}$ is closed in $\Gamma$ and $\Gamma$ is compact, $Y^{\prime}$ is compact as well.

Since $f$ is continuous, for every $\varepsilon>0$ and every $\tau \in Y^{\prime}$, there is an open $\operatorname{arc} \gamma(\tau) \ni \tau$ of the length $|\gamma(\tau)|<\varepsilon$ and such that $f(t)>0$ for all $t \in \overline{\gamma(\tau)}$. Without loss of generality, we can choose endpoints of $\gamma(\tau)$ such that either the right (left) half-neighborhood of the point $\tau$ is separated from $Y \backslash\{\tau\}$ or the right (left) endpoint of the $\operatorname{arc} \gamma(\tau)$ belongs to $Y$ (in the second case the point $\tau$ is a limit point of the subset of $Y$, which lies from the right (from the left) of the point $\tau$ ).

As $Y^{\prime}$ is a compact set, from the open overlapping $\left\{\gamma(\tau): \tau \in Y^{\prime}\right\}$ we may extract a finite overlapping $\left\{\gamma\left(\tau_{k}\right): k=1,2, \ldots, n\right\}$. From each $\operatorname{arc} \gamma\left(\tau_{k}\right)$ we delete half-neighborhoods of $\tau_{k}$ which wholly lie in $\Lambda$ or in $(\Gamma \backslash \Lambda) \cup\left\{\tau_{k}\right\}$. Since the point $\tau_{k}$ is not an isolated point of the set $Y$, we can delete at most one neighborhood of $\tau_{k}$. Thus, from each $\operatorname{arc} \gamma\left(\tau_{k}\right)$ we obtain an $\operatorname{arc} u\left(\tau_{k}\right)$, endpoints of which belong to $Y$, and

$$
\left(u\left(\tau_{k}\right) \cap Y\right) \cup\left\{\tau_{k}\right\}=\gamma\left(\tau_{k}\right) \cap Y .
$$

From (2.15) we see that the set

$$
\left(\Gamma \backslash\left(\bigcup_{k=1}^{n} \gamma\left(\tau_{k}\right)\right)\right) \cap Y=\left(\Gamma \backslash\left(\bigcup_{k=1}^{n} u\left(\tau_{k}\right)\right)\right) \cap Y
$$

is empty or finite.

We consecutively exclude from the set $\left\{u\left(\tau_{k}\right): k=1,2, \ldots, n\right\}$ the $\operatorname{arcs} u\left(\tau_{k}\right)$ contained in the union of the remaining arcs. After that we obtain a system of arcs such that every two arcs either are not intersected, or are intersected but each arc does not contain the other one. Moreover, the intersection of any three such arcs is empty. Without loss of generality, we assume that the original system $\left\{u\left(\tau_{k}\right): k=1,2, \ldots, n\right\}$ has this property. In that case there are pairwise disjoint open $\operatorname{arcs} v_{k}, k=1,2, \ldots, n$, with endpoints in $Y$, such that $v_{k} \subset u\left(\tau_{k}\right)$ and

$$
\bigcup_{k=1}^{n} \overline{v_{k}}=\bigcup_{k=1}^{n} \overline{u\left(\tau_{k}\right)} \supset Y^{\prime}
$$

Clearly, all $\operatorname{arcs} v_{k}, k=1,2, \ldots, n$, are $\alpha_{m}$-invariant, and for every arc $v_{k}$,

$$
f(t)>0 \quad \text { for all } \quad t \in \overline{v_{k}}, \quad \overline{v_{k}} \cap Y^{\prime} \neq \emptyset .
$$

Further, if the set

$$
v_{0}:=\Gamma \backslash\left(\bigcup_{k=1}^{n} \overline{v_{k}}\right)
$$


is empty, then we have the desired decomposition of $\Gamma$.

Otherwise, the open set $v_{0}$ is non-empty and $\alpha_{m}$-invariant. From (2.16) we see that

$$
v_{0} \cap Y=\left(\Gamma \backslash\left(\bigcup_{k=1}^{n} \overline{u\left(\tau_{k}\right)}\right)\right) \cap\left(Y \backslash Y^{\prime}\right),
$$

that is, the set $v_{0} \cap Y$ is finite.

Since the open set $v_{0}$ is not empty, the set

$$
v_{0} \cap(\Gamma \backslash Y)=\left(v_{0} \cap(\Gamma \backslash \Phi)\right) \cup\left(v_{0} \cap(\Phi \backslash \Lambda)\right)
$$

consists of a finite set of open arcs with endpoints in $v_{0} \cap Y$. Denoting these arcs by $\omega_{i}$ and $\gamma_{j}$ if they lie, respectively, in $\Gamma \backslash \Phi$ and $\Phi \backslash \Lambda$, we obtain

$$
v_{0} \cap(\Gamma \backslash \Phi)=\bigcup_{i} \omega_{i}, \quad v_{0} \cap(\Phi \backslash \Lambda)=\bigcup_{j} \gamma_{j} .
$$

Clearly, these arcs are $\alpha_{m}$-invariant, and we get the desired decomposition (2.14).

Proposition 2.6. The set $Y \backslash Y^{\prime}$ of all isolated points of $Y$ is contained in the set of all endpoints of connected components $\gamma \subset \Phi \backslash \Lambda(=\Gamma \backslash \Lambda)$.

The proof is obvious.

\section{Two-sided invertibility: sufficient conditions}

\subsection{Estimate for the spectral radius of weighted shift operators}

In Sections 3 and 4 we suppose that $\Gamma$ is an oriented Jordan smooth curve and $\alpha$ is an orientation preserving diffeomorphism of $\Gamma$ onto itself which has an arbitrary nonempty set $\Lambda$ of fixed points. We equip $\Gamma$ with the Lebesgue length measure $|d \tau|$. In the following we will consider all rearrangement-invariant spaces over the finite measure space $(\Gamma,|d \tau|)$. Also we always suppose that r.-i. spaces $X(\Gamma)$ have nontrivial Boyd indices $\alpha_{X}, \beta_{X}$. In that case the operator $A=a I-b W$ is bounded in the space $X(\Gamma)$, due to the Boyd interpolation theorem [8].

If $\Omega$ is a subset of $\Gamma$ of a positive measure, then we will denote by $X(\Omega)$ the subspace of $X(\Gamma)$ which consists of functions supported in $\Omega$. In view of Lemma 2.3, $X(\Omega)$ is an r.-i. space with the same Boyd and Zippin indices as the whole space $X(\Gamma)$.

Denote by $C(\Gamma)$ the set of all continuous functions on $\Gamma$.

Theorem 3.1. The spectral radius of the weighted shift operator $g W$ with coefficient (weight) $g \in C(\Gamma)$ in the Lebesgue space $L^{p}(\Gamma), 1<p<\infty$, is calculated by the formula

$$
r\left(g W ; L^{p}(\Gamma)\right)=\max _{\tau \in \Lambda}|g(\tau)|\left|\alpha^{\prime}(\tau)\right|^{-1 / p} .
$$

This theorem follows from the results of [15]. For further generalizations of the formula for the spectral radius of the weighted shift operator, see [2, Ch. 1, Section 5]. 
Theorem 3.2. The spectral radius of the weighted shift operator $g W$ with coefficient (weight) $g \in C(\Gamma)$ in an r.-i. space $X(\Gamma)$ with nontrivial Boyd indices $\alpha_{X}, \beta_{X}$ satisfies the estimate

$$
r(g W ; X(\Gamma)) \leq \max _{\tau \in \Lambda}\left(|g(\tau)| \max \left\{\left|\alpha^{\prime}(\tau)\right|^{-\alpha_{X}},\left|\alpha^{\prime}(\tau)\right|^{-\beta_{X}}\right\}\right) .
$$

Proof. By Theorem 3.1, the spectral radii of the operator $g W$ in the Lebesgue spaces $L^{1 / \alpha_{X}}(\Gamma)$ and $L^{1 / \beta_{X}}(\Gamma)$, are calculated by

$$
r\left(g W ; L^{1 / \alpha_{X}}(\Gamma)\right)=\max _{\tau \in \Lambda}|g(\tau)|\left|\alpha^{\prime}(\tau)\right|^{-\alpha_{X}}, \quad r\left(g W ; L^{1 / \beta_{X}}(\Gamma)\right)=\max _{\tau \in \Lambda}|g(\tau)|\left|\alpha^{\prime}(\tau)\right|^{-\beta_{X}},
$$

respectively. Theorem 2.4 and the latter equalities give

$$
\begin{aligned}
r(g W ; X(\Gamma)) & \leq \max \left\{r\left(g W ; L^{1 / \alpha_{X}}(\Gamma)\right), r\left(g W ; L^{1 / \beta_{X}}(\Gamma)\right)\right\} \\
& =\max \left\{\max _{\tau \in \Lambda}|g(\tau)|\left|\alpha^{\prime}(\tau)\right|^{-\alpha_{X}}, \max _{\tau \in \Lambda}|g(\tau)|\left|\alpha^{\prime}(\tau)\right|^{-\beta_{X}}\right\} \\
& =\max _{\tau \in \Lambda}\left(|g(\tau)| \max \left\{\left|\alpha^{\prime}(\tau)\right|^{-\alpha_{X}},\left|\alpha^{\prime}(\tau)\right|^{-\beta_{X}}\right\}\right) .
\end{aligned}
$$

\subsection{Sufficient conditions for the two-sided invertibility}

For the functional operator (1.1) define the two continuous functions $\eta_{i}: \Gamma \rightarrow \mathbf{R}, i \in\{0,1\}$, by the formulas

$$
\begin{aligned}
\eta_{0}(t) & :=|a(t)|-|b(t)| \min \left\{\left|\alpha^{\prime}(t)\right|^{-\alpha_{X}},\left|\alpha^{\prime}(t)\right|^{-\beta_{X}}\right\} \\
\eta_{1}(t) & :=|a(t)|-|b(t)| \max \left\{\left|\alpha^{\prime}(t)\right|^{-\alpha_{X}},\left|\alpha^{\prime}(t)\right|^{-\beta_{X}}\right\} .
\end{aligned}
$$

Lemma 3.3. Let $\gamma$ be a closed $\alpha$-invariant arc of $\Gamma$.

(a) If $\eta_{0}(t)<0$ for every $t \in \gamma$, then the operator $A$ is two-sided invertible in $X(\gamma)$, and

$$
A^{-1}=-W^{-1} \sum_{n=0}^{\infty}\left(b^{-1} a W^{-1}\right)^{n} b^{-1} I .
$$

(b) If $\eta_{1}(t)>0$ for every $t \in \gamma$, then the operator $A$ is two-sided invertible in $X(\gamma)$, and

$$
A^{-1}=\sum_{n=0}^{\infty}\left(a^{-1} b W\right)^{n} a^{-1} I .
$$

Proof. We consider the more difficult part (a). From the definition of $\eta_{0}$ we see that if $\eta_{0}(t)<0$ for all $t \in \gamma$, then

$$
0 \leq|a(t)|<|b(t)| \min \left\{\left|\alpha^{\prime}(t)\right|^{-\alpha_{X}},\left|\alpha^{\prime}(t)\right|^{-\beta_{X}}\right\}, \quad t \in \gamma .
$$

Hence, $b$ is invertible in $C(\gamma)$. In that case

$$
A=-b\left(I-b^{-1} a W^{-1}\right) W
$$


Let us show the invertibility of the factor $I-b^{-1} a W$. For fixed points of $\alpha$ we get $\alpha_{-1}^{\prime}(\tau)=$ $1 / \alpha^{\prime}\left[\alpha_{-1}(\tau)\right]=1 / \alpha^{\prime}(\tau)$. Then, taking into account that $W_{\alpha}^{-1}=W_{\alpha_{-1}}$ and the arc $\gamma$ is $\alpha$-invariant, we infer from Theorem 3.2 that

$$
\begin{aligned}
r\left(b^{-1} a W^{-1} ; X(\gamma)\right) & \leq \max _{\tau \in \Lambda \cap \gamma}\left(|b(\tau)|^{-1}|a(\tau)| \max \left\{\left|\alpha_{-1}^{\prime}(\tau)\right|^{-\alpha_{X}},\left|\alpha_{-1}^{\prime}(\tau)\right|^{-\beta_{X}}\right\}\right) \\
& =\max _{\tau \in \Lambda \cap \gamma}\left(|a(\tau)|\left(|b(\tau)| \min \left\{\left|\alpha^{\prime}(\tau)\right|^{-\alpha_{X}},\left|\alpha^{\prime}(\tau)\right|^{-\beta_{X}}\right\}\right)^{-1}\right) .
\end{aligned}
$$

From (3.4) and (3.6) we see that $r\left(b^{-1} a W^{-1} ; X(\gamma)\right)<1$. Hence, the operator $I-b^{-1} a W^{-1}$ is invertible in $X(\gamma)$, and

$$
\left(I-b^{-1} a W^{-1}\right)^{-1}=\sum_{n=0}^{\infty}\left(b^{-1} a W^{-1}\right)^{n} .
$$

From (3.5), (3.7) and the invertibility of operators $b I$ and $W$ we conclude that $A$ is invertible and (3.3) holds. The assertion (b) can be proved analogously.

\section{One-sided invertibility: case of fixed points}

\subsection{Case of only two fixed points}

Let $X(\Gamma)$ be a reflexive r.-i. space of fundamental type with nontrivial Boyd indices, i.e., Boyd and Zippin indices satisfy the inequalities

$$
0<\alpha_{X}=p_{X} \leq q_{X}=\beta_{X}<1 \text {. }
$$

We say that an $\alpha$-invariant set $\Omega \subset \Gamma$ satisfies, respectively, the condition

$$
\begin{aligned}
R(\Omega) \text { if } & \text { for every } t \in \Omega \text { there exists a } k_{0}=k_{0}(t) \in \mathbf{Z} \text { such that } \\
& a\left[\alpha_{k}(t)\right] \neq 0 \text { for } k<k_{0} \quad \text { and } b\left[\alpha_{k}(t)\right] \neq 0 \text { for } k \geq k_{0}, \\
L(\Omega) \quad \text { if } \quad \text { for every } t \in \Omega \text { there exists a } k_{0}=k_{0}(t) \in \mathbf{Z} \text { such that } & \\
& a\left[\alpha_{k}(t)\right] \neq 0 \text { for } k>k_{0} \quad \text { and } \quad b\left[\alpha_{k}(t)\right] \neq 0 \text { for } k<k_{0} .
\end{aligned}
$$

Denote by $G C(\Omega)$ the set of all functions invertible in $C(\Omega)$, that is, the set of all functions $a \in C(\Omega)$ such that

$$
\inf _{t \in \Omega}|a(t)|>0
$$

In the case of only two fixed points we obtain from [13, Theorem 6.8] and Lemma 2.3 the following result.

Theorem 4.1. Let $\gamma \subset \Gamma$ be an open arc with endpoints $\tau_{ \pm}$and $\Lambda \cap \bar{\gamma}=\left\{\tau_{-}, \tau_{+}\right\}$. The operator $A$ is right (left) invertible in the subspace $X(\gamma)$ if and only if one of the three conditions holds:

$$
\begin{array}{lll}
\eta_{1}\left(\tau_{-}\right)>0, & \eta_{1}\left(\tau_{+}\right)>0, & a \in G C(\bar{\gamma}) \\
\eta_{0}\left(\tau_{-}\right)<0, & \eta_{0}\left(\tau_{+}\right)<0, & b \in G C(\bar{\gamma})
\end{array}
$$




$$
\eta_{0}\left(\tau_{+}\right)<0<\eta_{1}\left(\tau_{-}\right) \text {and } R(\gamma) \text { is fulfilled }
$$

(respectively, (4.1), (4.2), or

$$
\left.\eta_{0}\left(\tau_{-}\right)<0<\eta_{1}\left(\tau_{+}\right) \text {and } L(\gamma) \text { is fulfilled }\right) .
$$

The restrictions $\alpha_{X}=p_{X}$ and $\beta_{X}=q_{X}$ are essential for the proof of this theorem given in [13]. From Theorem 4.1 and the inequality $\eta_{1}(t) \leq \eta_{0}(t), t \in \Gamma$, it follows

Corollary 4.2. If the operator $A$ is one-sided invertible in the subspace $X(\gamma)$, then

$$
\eta_{0}(\tau) \eta_{1}(\tau)>0, \quad \tau \in\left\{\tau_{-}, \tau_{+}\right\}
$$

Corollary 4.3. The intersection of the left and right spectra of the shift operator $W$ in the subspace $X(\gamma)$, that is, the set

$$
\sigma_{0}(W):=\{\lambda \in \mathbf{C}: \lambda I-W \quad \text { is not right and not left invertible in } X(\gamma)\},
$$

is given by the formula

$$
\sigma_{0}(W)=\bigcup_{\tau \in\left\{\tau_{-}, \tau_{+}\right\}}\{\lambda \in \mathbf{C}: \delta(\tau) \leq|\lambda| \leq \Delta(\tau)\}
$$

where $\delta(\tau):=\min \left\{\left|\alpha^{\prime}(\tau)\right|^{-\alpha_{X}},\left|\alpha^{\prime}(\tau)\right|^{-\beta_{X}}\right\}, \quad \Delta(\tau):=\max \left\{\left|\alpha^{\prime}(\tau)\right|^{-\alpha_{X}},\left|\alpha^{\prime}(\tau)\right|^{-\beta_{X}}\right\}$.

Proof. By Corollary 4.2, if the operator $\lambda I-W$ is one-sided invertible in $X(\gamma)$, then

$$
\eta_{0}(\tau) \eta_{1}(\tau)=(|\lambda|-\delta(\tau))(|\lambda|-\Delta(\tau))>0 \quad \text { for } \quad \tau \in\left\{\tau_{-}, \tau_{+}\right\}
$$

or, equivalently,

$$
|\lambda| \notin[\delta(\tau), \Delta(\tau)] \quad \text { for } \quad \tau \in\left\{\tau_{-}, \tau_{+}\right\} .
$$

On the other hand, if (4.4) does not hold, then one of the following four conditions is satisfied:

1) $|\lambda|<\min \left\{\delta\left(\tau_{-}\right), \delta\left(\tau_{+}\right)\right\} \Longleftrightarrow \eta_{0}\left(\tau_{-}\right)<0$ and $\eta_{0}\left(\tau_{+}\right)<0$,

2) $|\lambda|>\max \left\{\Delta\left(\tau_{-}\right), \Delta\left(\tau_{+}\right)\right\} \Longleftrightarrow \eta_{1}\left(\tau_{-}\right)>0$ and $\eta_{1}\left(\tau_{+}\right)>0$,

3) $\Delta\left(\tau_{-}\right)<|\lambda|<\delta\left(\tau_{+}\right) \quad \Longleftrightarrow \eta_{0}\left(\tau_{+}\right)<0<\eta_{1}\left(\tau_{-}\right)$,

4) $\Delta\left(\tau_{+}\right)<|\lambda|<\delta\left(\tau_{-}\right) \quad \Longleftrightarrow \eta_{0}\left(\tau_{-}\right)<0<\eta_{1}\left(\tau_{+}\right)$.

Since $\lambda \neq 0$ in the cases 2)-4), we infer from Theorem 4.1 that the operator $\lambda I-W$ is two-sided invertible in $X(\gamma)$ in cases 1$)-2$ ), right invertible in case 3 ), and left invertible in case 4$)$.

Thus, the operator $\lambda I-W$ is one-sided invertible in the subspace $X(\gamma)$ if and only if (4.4) holds. Hence, $\lambda I-W$ is neither left invertible, nor right invertible in the subspace $X(\gamma)$ if and only if $\lambda \in \sigma_{0}(W)$.

Clearly, if $\alpha_{X}<\beta_{X}$, then $\sigma_{0}$ is the union of two annuli which degenerate in two circles whenever $\alpha_{X}=\beta_{X}$. Thus, in the case of arbitrary r.-i. spaces, in contrast to Lebesgue spaces, the following new quality appears: the intersection of the left and right spectra of the shift operator $W$ becomes massive (i.e., admits non-zero plain measure) in general. 


\subsection{Case of arbitrary nonempty set of fixed points}

In this subsection we prove a criterion for one-sided invertibility of the operator $A$ when the shift $\alpha$ has an arbitrary nonempty set of fixed points. In the proof we essentially use a decomposition of $\Gamma$ into a union of arcs of three types (see Lemma 2.5). Since these arcs are invariant with respect to the shift $\alpha$, we can check one-sided invertibility of $A$ in each subspace of functions supported in these arcs.

Theorem 4.4. The operator $A$ is right (left) invertible in the space $X(\Gamma)$ if and only if the following three conditions simultaneously hold:

(i) the operator $A$ is right (left) invertible in $X(\gamma)$ for every connected component $\gamma \subset \Phi \backslash \Lambda$;

(ii) $a(t) \neq b(t)$ for every $t \in \Gamma \backslash \Phi$;

(iii) if the set $Y$ is infinite, then $\eta_{0}(\tau) \eta_{1}(\tau)>0$ for every $\tau \in Y^{\prime}$.

Proof. Necessity. Suppose the operator $A$ is right (left) invertible in $X(\Gamma)$. Since the set $\Gamma \backslash \Phi$ and every connected component $\gamma \subset \Phi \backslash \Lambda$ are $\alpha$-invariant, the operator $A$ is bounded and right (left) invertible in every subspace $X(\gamma), \gamma \subset \Phi \backslash \Lambda$, and in the subspace $X(\Gamma \backslash \Phi)$. So, we get (i).

Since $\Gamma \backslash \Phi=\{t \in \Gamma \backslash Y: \alpha(t)=t\}$, we have $A=(a-b) I$ in the subspace $X(\Gamma \backslash \Phi)$. In view of the one-sided invertibility of $(a-b) I$, we obtain (ii). If the set $Y$ is finite, the necessity is proved.

Now we prove (iii) when the set $Y$ is infinite. Assume the contrary: $\eta_{0}\left(\tau_{0}\right) \eta_{1}\left(\tau_{0}\right) \leq 0$ for some point $\tau_{0} \in Y^{\prime}$. In view of the stability of one-sided invertibility of an operator under small (in the operator norm) perturbations (see, e.g., [11, Ch. 2, Theorem 5.4]), there are $\widetilde{a}, \widetilde{b} \in C(\Gamma)$ such that the operator $\widetilde{a} I-\widetilde{b} W$ is right (left) invertible in $X(\Gamma)$, and at some point $\tau \in Y \backslash Y^{\prime}$, which is sufficiently close to $\tau_{0}$, the following inequality holds: $\widetilde{\eta}_{0}(\tau) \widetilde{\eta}_{1}(\tau) \leq 0$. Here quantities $\widetilde{\eta}_{0}$ and $\widetilde{\eta}_{1}$ are defined for the operator $\widetilde{a} I-\widetilde{b} W$ by formulas (3.1) and (3.2). Proposition 2.6 tells us that $\tau$ is an endpoint of some connected component $\gamma \subset \Phi \backslash \Lambda$. From Corollary 4.2 we obtain that the operator $\widetilde{a} I-\widetilde{b} W$ is not one-sided invertible in $X(\gamma)$. This contradicts to condition (i) just proved. Hence, condition (iii) is fulfilled. Necessity is proved.

Sufficiency. Since by (i) the operator $A$ is right (left) invertible in $X(\gamma)$ for every connected component $\gamma \subset \Phi \backslash \Lambda$, it follows from Corollary 4.2 that $\eta_{0}(\tau) \eta_{1}(\tau)>0$ for each endpoint $\tau$ of every such $\gamma$. Hence, by Proposition 2.6,

$$
\eta_{0}(\tau) \eta_{1}(\tau)>0 \quad \text { for every } \quad \tau \in Y \backslash Y^{\prime}
$$

which together with (iii) gives

$$
\eta_{0}(\tau) \eta_{1}(\tau)>0 \quad \text { for every } \quad \tau \in Y
$$

Since $\alpha^{\prime}(\tau)=1$ in every endpoint $\tau$ of every connected component $\omega \subset \Gamma \backslash \Phi$, we infer from (3.1)-(3.2) and (4.5) that $a(\tau) \neq b(\tau)$ for all those points $\tau$. Then in view of (ii), for every connected component $\omega \subset \Gamma \backslash \Phi$,

$$
a(t) \neq b(t) \quad \text { for every } \quad t \in \bar{\omega} \text {. }
$$


If the set $Y$ is finite, then condition (iii) disappears. Moreover, by Lemma 2.5(a) there is a finite decomposition

$$
\Gamma=\left(\bigcup_{i} \overline{\omega_{i}}\right) \cup\left(\bigcup_{j} \overline{\gamma_{j}}\right)
$$

where $\omega_{i} \subset \Gamma \backslash \Phi$ and $\gamma_{j} \subset \Phi \backslash \Lambda$ are pairwise disjoint, $\alpha$-invariant open arcs with endpoints in $Y$. Decompose the space $X(\Gamma)$ into the (finite) direct sum of its subspaces:

$$
X(\Gamma)=\left(\bigoplus_{i} X\left(\omega_{i}\right)\right) \oplus\left(\bigoplus_{j} X\left(\gamma_{j}\right)\right) .
$$

Clearly, the operator $A$ acts in each subspace of the decomposition (4.7). Since $\omega_{i} \subset \Gamma \backslash \Phi=$ $\{t \in \Gamma \backslash Y: \alpha(t)=t\}$, from (4.6) we see that the operator $A=(a-b) I$ is invertible in each subspace $X\left(\omega_{i}\right)$. Hence, taking into account condition (i), we obtain that the operator $A$ is right (left) invertible in the space $X(\Gamma)$. Thus, in the case of finite $Y$ sufficiency is proved.

Now we consider the case of infinite set $Y$. Since the functions $\eta_{0}$ and $\eta_{1}$ are continuous on $\Gamma$, from Lemma 2.5(b) and condition (iii) it follows that there is a finite decomposition

$$
\Gamma=\left(\bigcup_{i} \overline{\omega_{i}}\right) \cup\left(\bigcup_{j} \overline{\gamma_{j}}\right) \cup\left(\bigcup_{k} \overline{v_{k}}\right),
$$

where $\omega_{i} \subset \Gamma \backslash \Phi, \gamma_{j} \subset \Phi \backslash \Lambda$ and $v_{k} \subset \Gamma$ are pairwise disjoint, $\alpha$-invariant open arcs with endpoints in $Y$. Moreover, $\overline{v_{k}} \cap Y^{\prime} \neq \emptyset$ and

$$
\eta_{0}(\tau) \eta_{1}(\tau)>0 \quad \text { for all } \quad t \in \overline{v_{k}}
$$

and all $\operatorname{arcs} v_{k}$. Decompose the space $X(\Gamma)$ into the (finite) direct sum of its subspaces:

$$
X(\Gamma)=\left(\bigoplus_{i} X\left(\omega_{i}\right)\right) \oplus\left(\bigoplus_{j} X\left(\gamma_{j}\right)\right) \oplus\left(\bigoplus_{k} X\left(v_{k}\right)\right) .
$$

Clearly, the operator $A$ acts in each subspace of the decomposition (4.9). From (4.8) we see that either $\eta_{0}(\tau) \geq \eta_{1}(\tau)>0$, or $\eta_{1}(\tau) \leq \eta_{0}(\tau)<0$ for every $\tau \in \overline{v_{k}}$. Hence, by Lemma 3.3 , the operator $A$ is invertible in each subspace $X\left(v_{k}\right)$. As in the previous case, we see that the operator $A$ is invertible in each subspace $X\left(\omega_{i}\right)$ and is right (left) invertible in each subspace $X\left(\gamma_{j}\right)$. Thus, taking into account (4.9), we see that the operator $A$ is right (left) invertible in the space $X(\Gamma)$.

\subsection{Another form of the criterion for one-sided invertibility}

In this subsection we reformulate results of the previous subsection in terms of a function, which controls the two- and one-sided invertibility of the operator $A$. We start with the following important property of fixed points. 
Lemma 4.5 (see [16, Ch. 1, Lemma 2]). Let $\tau_{1}, \tau_{2} \in \Gamma$ be fixed points of an orientation preserving shift $\alpha$. If the arc $\left(\tau_{1}, \tau_{2}\right)$ does not contain fixed points of the shift $\alpha$, then for each point $t \in\left(\tau_{1}, \tau_{2}\right)$ the iterative sequence $\alpha_{n}(t)\left(\alpha_{-n}(t)\right)$ converges to a fixed point, either to the point $\tau_{1}$ (respectively, $\tau_{2}$ ) or to the point $\tau_{2}$ (respectively, $\tau_{1}$ ) independently of $t$.

In accordance with this property we define the following functions:

$$
\eta_{i}^{ \pm}(t):=\lim _{n \rightarrow \pm \infty} \eta_{i}\left[\alpha_{n}(t)\right], \quad t \in \Gamma, \quad i \in\{0,1\} .
$$

In view of Lemma 4.5 and the continuity of $a, b, \alpha^{\prime}$, the limits in (4.10) exist for every $t \in \Gamma$. Hence the functions $\eta_{i}^{ \pm}$are well-defined. From the definition of fixed points we see that

$$
\eta_{i}(\tau)=\eta_{i}^{+}(\tau)=\eta_{i}^{-}(\tau), \quad \tau \in \Lambda, \quad i \in\{0,1\} .
$$

For the functional operator (1.1), define five sets: $\Gamma_{1}:=\Gamma \backslash \Phi$,

$$
\begin{array}{ll}
\Gamma_{2}:=\left\{t \in \Phi: \eta_{1}^{-}(t)>0, \eta_{1}^{+}(t)>0\right\}, & \Gamma_{3}:=\left\{t \in \Phi: \eta_{0}^{-}(t)<0, \eta_{0}^{+}(t)<0\right\}, \\
\Gamma_{4}:=\left\{t \in \Phi: \eta_{0}^{+}(t)<0<\eta_{1}^{-}(t)\right\}, & \Gamma_{5}:=\left\{t \in \Phi: \eta_{0}^{-}(t)<0<\eta_{1}^{+}(t)\right\} .
\end{array}
$$

Clearly, these sets are pairwise disjoint and $\alpha$-invariant. Moreover, we infer from (4.11), the inequality $\eta_{1}(\tau) \leq \eta_{0}(\tau)$ and the definitions of $\Gamma_{4}$ and $\Gamma_{5}$, that

$$
\Gamma_{4} \cap \Lambda=\Gamma_{5} \cap \Lambda=\emptyset .
$$

Indeed, if, for example, $\tau \in \Gamma_{4} \cap \Lambda$, then $\eta_{1}^{+}(\tau) \leq \eta_{0}^{+}(\tau)<0<\eta_{1}^{-}(\tau) \leq \eta_{0}^{-}(\tau)$, which is impossible due to (4.11). Define the function

$$
\sigma_{A}(t):=\left\{\begin{array}{cl}
a(t)-b(t), & t \in \Gamma_{1}, \\
a(t), & t \in \Gamma_{2}, \\
-b(t), & t \in \Gamma_{3}, \\
0, & t \in \Gamma \backslash\left(\Gamma_{1} \cup \Gamma_{2} \cup \Gamma_{3}\right) .
\end{array}\right.
$$

Now reformulate Theorem 4.4 in terms of the function $\sigma_{A}$ and sets $\Gamma_{j}, j \in\{1,2, \ldots, 5\}$.

Theorem 4.6. The operator $A$ is right (left) invertible in the space $X(\Gamma)$ if and only if

$$
\sigma_{A}(t) \neq 0 \text { for all } t \in \Gamma \backslash \Gamma_{4} \text { and } R\left(\Gamma_{4}\right) \text { holds }
$$

(respectively,

$$
\left.\sigma_{A}(t) \neq 0 \text { for all } t \in \Gamma \backslash \Gamma_{5} \quad \text { and } \quad L\left(\Gamma_{5}\right) \text { holds }\right) .
$$

Proof. Necessity. Let the operator $A$ be one-sided invertible in the space $X(\Gamma)$. Then conditions (i)-(iii) of Theorem 4.4 are satisfied. The proof of sufficiency in that theorem shows that conditions (i) and (iii) imply (4.5). Hence, for $\tau \in Y$, either $\eta_{0}(\tau) \geq \eta_{1}(\tau)>0$, or $\eta_{1}(\tau) \leq \eta_{0}(\tau)<0$, which gives $Y \subset \Gamma_{2} \cup \Gamma_{3}$. Taking into account (3.2) and (4.11), we conclude that in the first case $\tau \in \Gamma_{2} \cap Y$ and then

$$
|a(\tau)|>|b(\tau)| \max \left\{\left|\alpha^{\prime}(\tau)\right|^{-\alpha_{X}},\left|\alpha^{\prime}(\tau)\right|^{-\beta_{X}}\right\} \geq 0 .
$$


Hence,

$$
\sigma_{A}(\tau) \neq 0 \quad \text { for all } \quad \tau \in \Gamma_{2} \cap Y .
$$

Analogously, in the second case $\tau \in \Gamma_{3} \cap Y$ and

$$
\sigma_{A}(\tau) \neq 0 \text { for all } \quad \tau \in \Gamma_{3} \cap Y .
$$

On the other hand, condition (ii) of Theorem 4.4 implies

$$
\sigma_{A}(t) \neq 0 \text { for all } t \in \Gamma_{1} .
$$

Consider the case of the right invertibility. By Theorem 4.4(i), the operator $A$ is right invertible in the subspace $X(\gamma)$ for every connected component $\gamma \subset \Phi \backslash \Lambda$. Hence, Theorem 4.1 yields that one of the three conditions holds:

$$
\begin{aligned}
& \gamma \subset(\Phi \backslash \Lambda) \cap \Gamma_{2} \text { and } \sigma_{A}(t) \neq 0, \quad t \in \gamma ; \\
& \gamma \subset(\Phi \backslash \Lambda) \cap \Gamma_{3} \text { and } \sigma_{A}(t) \neq 0, \quad t \in \gamma ; \\
& \gamma \subset \Gamma_{4} \text { and } R(\gamma) \text { is fulfilled. }
\end{aligned}
$$

Clearly, $\gamma \cap \Gamma_{5}=\emptyset$. Thus,

$$
\Gamma=\Gamma_{1} \cup \Gamma_{2} \cup \Gamma_{3} \cup \Gamma_{4}, \quad Y \subset \Gamma_{2} \cap \Gamma_{3}, \quad \Gamma_{5}=\emptyset,
$$

and (4.17)-(4.21) imply that $\sigma_{A}(t) \neq 0$ for all $t \in \Gamma \backslash \Gamma_{4}=\Gamma_{1} \cup \Gamma_{2} \cup \Gamma_{3}$. Moreover, from (4.22) we see that $R\left(\Gamma_{4}\right)$ holds.

Analogously, in the case of the left invertibility we have

$$
\Gamma=\Gamma_{1} \cup \Gamma_{2} \cup \Gamma_{3} \cup \Gamma_{5}, \quad Y \subset \Gamma_{2} \cap \Gamma_{3}, \quad \Gamma_{4}=\emptyset,
$$

$\sigma_{A}(t) \neq 0$ for all $t \in \Gamma \backslash \Gamma_{5}=\Gamma_{1} \cup \Gamma_{2} \cup \Gamma_{3}$ and $L\left(\Gamma_{5}\right)$ holds. Necessity is proved.

Sufficiency. Suppose (4.16) is fulfilled. Since $\sigma_{A}(t) \neq 0$ for all $t \in \Gamma \backslash \Gamma_{5}$, we see that $\Gamma \backslash \Gamma_{5}=\Gamma_{1} \cup \Gamma_{2} \cup \Gamma_{3}$. Then from (4.14) and the definition of $\Gamma_{1}$ we infer that $Y \subset \Gamma_{2} \cup \Gamma_{3}$. If $\tau \in Y \cap \Gamma_{2}$, then $\eta_{0}(\tau) \geq \eta_{1}(\tau)>0$. Analogously, $\eta_{1}(\tau) \leq \eta_{0}(\tau)<0$ whenever $\tau \in Y \cap \Gamma_{3}$. Hence, $\eta_{0}(\tau) \eta_{1}(\tau)>0$ for all $\tau \in Y$. In particular, if the set $Y$ is infinite, then taking into account the embedding $Y^{\prime} \subset Y$, we get condition (iii) of Theorem 4.4. The condition $\sigma_{A}(t) \neq 0$ for all $t \in \Gamma_{1}$, implies condition (ii) of Theorem 4.4 .

From Lemma 4.5 it follows that the set $(\Phi \backslash \Lambda) \cap \Gamma_{2},\left((\Phi \backslash \Lambda) \cap \Gamma_{3},(\Phi \backslash \Lambda) \cap \Gamma_{5}\right)$ consists of at most countable union of connected components (open arcs), every of which wholly lies in $(\Phi \backslash \Lambda) \cap \Gamma_{2}$ (respectively, in $\left.(\Phi \backslash \Lambda) \cap \Gamma_{3},(\Phi \backslash \Lambda) \cap \Gamma_{5}\right)$. Moreover, each such $\operatorname{arc} \gamma$ does not contain fixed points of the shift $\alpha$, and the endpoints of this arc lie in $Y$. Hence, for every such arc we can apply Theorem 4.1 (case (4.1), (4.2), (4.3), respectively). By Theorem 4.1, the operator $A$ is left invertible in the subspace $X(\gamma)$ for every connected component $\gamma$ of the set

$$
\Phi \backslash \Lambda=\left((\Phi \backslash \Lambda) \cap \Gamma_{2}\right) \cup\left((\Phi \backslash \Lambda) \cap \Gamma_{3}\right) \cup\left((\Phi \backslash \Lambda) \cap \Gamma_{5}\right) .
$$

Hence, condition (i) of Theorem 4.4 is satisfied along with conditions (ii) and (iii). Therefore, we obtain the required result from Theorem 4.4.

Analogously, one can derive that (4.15) implies the right invertibility of $A$. 


\section{One-sided invertibility: general case}

\subsection{Main result}

In this section we generalize results of the previous section to the case of shifts having arbitrary nonempty sets of periodic points. Let $\Gamma$ be an oriented Jordan smooth curve. Suppose $\alpha$ is a diffeomorphism of $\Gamma$ onto itself which preserves or changes the orientation on $\Gamma$ and has an arbitrary nonempty set $\Lambda$ of periodic points of multiplicity $m \geq 1$ if $\alpha$ preserves the orientation on $\Gamma$, and the multiplicity $m=2$ (for all points of $\Lambda$ except for two fixed points of $\alpha$ ) if $\alpha$ changes the orientation on $\Gamma$. Suppose $X(\Gamma)$ is a reflexive r.-i. space of fundamental type with nontrivial Boyd indices.

For a continuous function $f: \Gamma \rightarrow \mathbf{C}$ and all $k \in \mathbf{N}$, we introduce the functions

$$
f_{k}(t):=\prod_{i=0}^{k-1} f\left[\alpha_{i}(t)\right], \quad t \in \Gamma
$$

This notation is consistent with the formula for the derivative of the shift $\alpha_{k}$ if $f=\alpha^{\prime}$.

Proposition 5.1. Suppose $\alpha$ is a shift having periodic points of multiplicity $m \geq 1$ if $\alpha$ preserves the orientation on $\Gamma$, and let $m=2$ if $\alpha$ changes the orientation on $\Gamma$. If $f: \Gamma \rightarrow \mathbf{C}$ is a continuous function, then for every $t \in \Gamma$ and every $k \in \mathbf{Z}$,

$$
\lim _{n \rightarrow \pm \infty} f_{m}\left[\alpha_{m n+k}(t)\right]=\lim _{n \rightarrow \pm \infty} f_{m}\left[\alpha_{m n}(t)\right] .
$$

Proof. Clearly, it is sufficient to consider the case $1 \leq k \leq m-1$. Then

$$
\left(\prod_{i=0}^{k-1} f\left[\alpha_{m n+i}(t)\right]\right) f_{m}\left[\alpha_{m n+k}(t)\right]=\left(\prod_{i=0}^{k-1} f\left[\alpha_{m(n+1)+i}(t)\right]\right) f_{m}\left[\alpha_{m n}(t)\right] .
$$

Since the shift $\alpha_{m}$ has only fixed points, Lemma 4.5 implies that for every $t \in \Gamma$, the sequence $\alpha_{m n}(t)$ converges to a fixed point of $\alpha_{m}$ as $n \rightarrow \pm \infty$. Passing to the limit in (5.2) as $n \rightarrow \pm \infty$, in view of the continuity of $f$, we get

$$
\left\{\prod_{i=0}^{k-1} l_{i}\right\} \cdot \lim _{n \rightarrow \pm \infty} f_{m}\left[\alpha_{m n+k}(t)\right]=\left\{\prod_{i=0}^{k-1} l_{i}\right\} \cdot \lim _{n \rightarrow \pm \infty} f_{m}\left[\alpha_{m n}(t)\right]
$$

where

$$
l_{i}:=f\left(\lim _{n \rightarrow \pm \infty} \alpha_{m n+i}(t)\right) .
$$

Moreover, since the factors belonging to the first product from the left (from the right) in (5.2) are contained in the second product from the right (from the left) in (5.2), we see that if $l_{i}=0$ for some $i=0, \ldots, k-1$, then

$$
\lim _{n \rightarrow \pm \infty} f_{m}\left[\alpha_{m n+k}(t)\right]=\lim _{n \rightarrow \pm \infty} f_{m}\left[\alpha_{m n}(t)\right]=0 .
$$

Otherwise, we divide equality (5.3) on the product $l_{0} l_{1} \cdots l_{k-1}$, which again gives (5.1). 
For the functional operator (1.1), in the case $m \geq 1$ define the two continuous functions $\eta_{i}: \Gamma \rightarrow \mathbf{R}, i \in\{0,1\}$, by the formulas

$$
\begin{aligned}
& \eta_{0}(t):=\left|a_{m}(t)\right|-\left|b_{m}(t)\right| \min \left\{\left|\alpha_{m}^{\prime}(t)\right|^{-\alpha_{X}},\left|\alpha_{m}^{\prime}(t)\right|^{-\beta_{X}}\right\}, \\
& \eta_{1}(t):=\left|a_{m}(t)\right|-\left|b_{m}(t)\right| \max \left\{\left|\alpha_{m}^{\prime}(t)\right|^{-\alpha_{X}},\left|\alpha_{m}^{\prime}(t)\right|^{-\beta_{X}}\right\} .
\end{aligned}
$$

Clearly, the shift $\alpha_{m}$ has only fixed points. Then for $m \geq 1$, we can define the following functions in correspondence with Lemma 4.5:

$$
\eta_{i}^{ \pm}(t):=\lim _{n \rightarrow \pm \infty} \eta_{i}\left[\alpha_{m n}(t)\right], \quad t \in \Gamma, \quad i \in\{0,1\}
$$

In view of Lemma 4.5 and the continuity of the functions $a_{m}, b_{m}, \alpha_{m}^{\prime}$, the limits in (5.6) exist for every $t \in \Gamma$. Hence, the functions $\eta_{i}^{ \pm}$are well-defined. Introduce the sets $\Gamma_{j}, j \in\{1,2, \ldots, 5\}$, as before, by formulas (4.12), (4.13).

Corollary 5.2. For every $i \in\{0,1\}$ and every $k \in \mathbf{Z}$,

$$
\eta_{i}^{-}(t)=\eta_{i}^{-}\left[\alpha_{k}(t)\right], \quad \eta_{i}^{+}(t)=\eta_{i}^{+}\left[\alpha_{k}(t)\right], \quad t \in \Gamma,
$$

and hence, the sets $\Gamma_{j}, j \in\{1,2, \ldots, 5\}$, are $\alpha_{k}$-invariant.

This statement follows from Proposition 5.1.

Consider the function

$$
\sigma_{A}(t):=\left\{\begin{array}{clrl}
a_{m}(t)-b_{m}(t), & t \in \Gamma_{1}, \\
a_{m}(t), & & t \in \Gamma_{2}, \\
-b_{m}(t), & t \in \Gamma_{3}, \\
0, & & t \in \Gamma \backslash\left(\Gamma_{1} \cup \Gamma_{2} \cup \Gamma_{3}\right) .
\end{array}\right.
$$

Obviously, the functions (5.4)-(5.7) generalize the corresponding functions, introduced in Section 4 , to the case of arbitrary multiplicity $m \geq 1$ of periodic points (recall that $m=2$ if $\alpha$ changes the orientation on $\Gamma$ ).

Now we are able to formulate the main result.

Theorem 5.3. Let $\Gamma$ be an oriented Jordan smooth curve and let $\alpha$ be a diffeomorphism of $\Gamma$ onto itself which has an arbitrary nonempty set of periodic points. The operator $A$ is right (left) invertible in a reflexive r.-i. space $X(\Gamma)$ of fundamental type with nontrivial Boyd indices if and only if

$$
\sigma_{A}(t) \neq 0 \text { for all } t \in \Gamma \backslash \Gamma_{4} \quad \text { and } \quad R\left(\Gamma_{4}\right) \text { holds }
$$

(respectively,

$$
\left.\sigma_{A}(t) \neq 0 \text { for all } t \in \Gamma \backslash \Gamma_{5} \quad \text { and } \quad L\left(\Gamma_{5}\right) \text { holds }\right) \text {. }
$$

For the proof of this theorem we need some auxiliary results, which will be stated in the next two subsections. 


\subsection{Adjoint operator}

Since the Lebesgue measure on $\Gamma$ is separable (see, e.g., [12], Ch. 1, Subsection 6.10]), from Lemma 2.1 it follows that the general form of a linear functional on the reflexive r.-i. space $X(\Gamma)$ is given by

$$
l(u)=(u, v):=\int_{\Gamma} u(\tau) \overline{v(\tau)}|d \tau|, \quad u \in X(\Gamma), \quad v \in X^{\prime}(\Gamma) .
$$

From (5.10) it follows that the adjoint operator $A^{*}$ acting on the space $X^{\prime}(\Gamma)=(X(\Gamma))^{*}$ and defined by the equality $(A u, v)=\left(u, A^{*} v\right)$, has the form

$$
A^{*}=\bar{a} I-\overline{b\left(\alpha_{-1}\right)}\left|\alpha_{-1}^{\prime}\right| W^{-1}
$$

(here and in what follows $f\left(\alpha_{k}\right)=f \circ \alpha_{k}, k \in \mathbf{Z}$ ).

It is easy to see that if $\tau$ is a periodic point of the multiplicity $m \geq 1$ for the shift $\alpha$, then $\tau$ is also a periodic point for the shift $\beta=\alpha_{-1}$ of the same multiplicity.

Since the operator $A^{*}$ has the same form as the operator $A$, for $A^{*}$ one can define functions $\widetilde{\eta}_{i}$ by analogy with (5.4) and (5.5), and functions $\widetilde{\eta}_{i}^{ \pm}$by analogy with (5.6), replacing the coefficients $a, b$, the shift $\alpha$, and the Boyd indices $\alpha_{X}, \beta_{X}$, respectively, by the coefficients $\bar{a}, \overline{b\left(\alpha_{-1}\right)}\left|\alpha_{-1}^{\prime}\right|$, the shift $\alpha_{-1}$, and the Boyd indices $\alpha_{X^{\prime}}, \beta_{X^{\prime}}$ of the associate (= dual) space $X^{\prime}(\Gamma)$.

Lemma 5.4. For every $t \in \Gamma$ and $i \in\{0,1\}$, we have

$$
\widetilde{\eta}_{i}^{+}(t)=\eta_{i}^{-}(t), \quad \widetilde{\eta}_{i}^{-}(t)=\eta_{i}^{+}(t) .
$$

Proof. Taking into account the relations

$$
\prod_{i=0}^{m-1} \alpha_{-1}^{\prime}\left[\alpha_{-i}(t)\right]=\alpha_{-m}^{\prime}(t)=\left(\alpha_{m}^{\prime}\left[\alpha_{-m}(t)\right]\right)^{-1}
$$

and the equalities (2.10), we infer from the definition of $\widetilde{\eta}_{0}$ that

$$
\begin{aligned}
\tilde{\eta}_{0}(t) & =\left|\prod_{i=0}^{m-1} \overline{a\left[\alpha_{-i}(t)\right]}\right|-\left|\prod_{i=0}^{m-1}\left(\overline{b\left[\alpha_{-i-1}(t)\right]} \cdot\left|\alpha_{-1}^{\prime}\left[\alpha_{-i}(t)\right]\right|\right)\right| \min \left\{\left|\alpha_{-m}^{\prime}(t)\right|^{\left.-\alpha_{X^{\prime}},\left|\alpha_{-m}^{\prime}(t)\right|^{-\beta_{X^{\prime}}}\right\}}\right. \\
& \left.\left.=\left|a_{m}\left[\alpha_{-m+1}(t)\right]\right|-\left.\left|b_{m}\left[\alpha_{-m}(t)\right]\right| \min \left\{\mid \alpha_{-m}^{\prime}(t)\right]\right|^{1-\alpha_{X^{\prime}}}, \mid \alpha_{-m}^{\prime}(t)\right]\left.\right|^{1-\beta_{X^{\prime}}}\right\} \\
& =\left|a_{m}\left[\alpha_{-m+1}(t)\right]\right|-\left|b_{m}\left[\alpha_{-m}(t)\right]\right| \min \left\{\left|\alpha_{m}^{\prime}\left[\alpha_{-m}(t)\right]\right|^{-\alpha_{X}},\left|\alpha_{m}^{\prime}\left[\alpha_{-m}(t)\right]\right|^{-\beta_{X}}\right\} \cdot
\end{aligned}
$$

Hence, from (5.13) we get

$$
\begin{aligned}
\widetilde{\eta}_{0}\left[\alpha_{m n}(t)\right] & =\left|a_{m}\left[\alpha_{m n-m+1}(t)\right]\right|-\left|b_{m}\left[\alpha_{m n-m}(t)\right]\right| \times \\
& \times \min \left\{\left|\alpha_{m}^{\prime}\left[\alpha_{m n-m}(t)\right]\right|^{-\alpha_{X}},\left|\alpha_{m}^{\prime}\left[\alpha_{m n-m}(t)\right]\right|^{-\beta_{X}}\right\} .
\end{aligned}
$$

Passing to the limit as $n \rightarrow+\infty$, we get from (5.14) and Proposition 5.1 that

$$
\widetilde{\eta}_{0}^{-}(t)=\lim _{n \rightarrow+\infty} \widetilde{\eta}_{0}\left[\alpha_{m n}(t)\right]=\lim _{n \rightarrow+\infty} \eta_{0}\left[\alpha_{m n}(t)\right]=\eta_{0}^{+}(t) .
$$


Analogously, one can prove that

$$
\tilde{\eta}_{0}^{+}(t)=\eta_{0}^{-}(t), \quad \tilde{\eta}_{1}^{-}(t)=\eta_{1}^{+}(t), \quad \tilde{\eta}_{1}^{+}(t)=\eta_{1}^{-}(t) .
$$

Put $\widetilde{\Phi}:=\overline{\left\{t \in \Gamma: \alpha_{-m}(t) \neq t\right\}}$. Clearly, $\widetilde{\Phi}=\Phi$. Consider the set $\widetilde{\Gamma}_{1}:=\Gamma \backslash \widetilde{\Phi}=$ $\Gamma \backslash \Phi=\Gamma_{1}$. With the help of functions $\widetilde{\eta}_{i}^{ \pm}$, we define the sets $\widetilde{\Gamma}_{j}, j \in\{2,3,4,5\}$, by analogy with (4.12), (4.13). From Lemma 5.4 we see that

$$
\widetilde{\Gamma}_{2}=\Gamma_{2}, \quad \widetilde{\Gamma}_{3}=\Gamma_{3}, \quad \widetilde{\Gamma}_{4}=\Gamma_{5}, \quad \widetilde{\Gamma}_{5}=\Gamma_{4} .
$$

For the adjoint operator (5.11), define the function $\sigma_{A^{*}}(t)$ and the conditions $\widetilde{R}(\Omega), \widetilde{L}(\Omega)$ by analogy with (5.7) and the conditions of $R(\Omega), L(\Omega)$, respectively, using the coefficients $\bar{a}$ and $\overline{b\left(\alpha_{-1}\right)}\left|\alpha_{-1}^{\prime}\right|$ instead of $a$ and $b$, the shift $\alpha_{-1}$ instead of $\alpha$, the sets $\widetilde{\Gamma}_{j}$ instead of $\Gamma_{j}$.

Proposition 5.5. (a) The conditions $L\left(\Gamma_{5}\right)$ and $\widetilde{R}\left(\widetilde{\Gamma}_{4}\right)$ are equivalent.

(b) The conditions $L\left(\Gamma_{4}\right)$ and $\widetilde{R}\left(\widetilde{\Gamma}_{5}\right)$ are equivalent.

The proof is straightforward in view of (5.15).

Lemma 5.6. (a) $\sigma_{A}(t) \neq 0$ for all $t \in \Gamma \backslash \Gamma_{5}$ if and only if $\sigma_{A^{*}}(t) \neq 0$ for all $t \in \Gamma \backslash \widetilde{\Gamma}_{4}$. (b) $\sigma_{A}(t) \neq 0$ for all $t \in \Gamma \backslash \Gamma_{4}$ if and only if $\sigma_{A^{*}}(t) \neq 0$ for all $t \in \Gamma \backslash \widetilde{\Gamma}_{5}$.

Proof. (a) Since $\alpha_{m}(t)=t$ for $t \in \Gamma_{1}$, we have from the first identity in (5.12) that

$$
\prod_{i=0}^{m-1} \alpha_{-1}^{\prime}\left[\alpha_{-i}(t)\right]=\alpha_{-m}^{\prime}(t)=1, \quad t \in \widetilde{\Gamma}_{1}=\Gamma_{1} .
$$

Hence, using again the identity $\alpha_{m}(t)=t$ we get

$$
\begin{aligned}
\sigma_{A^{*}}(t) & \left.=\prod_{i=0}^{m-1} \overline{a\left[\alpha_{-i}(t)\right]}-\prod_{i=0}^{m-1} \overline{\left(b\left[\alpha_{-i-1}(t)\right]\right.} \cdot\left|\alpha_{-1}^{\prime}\left[\alpha_{-i}(t)\right]\right|\right)=\prod_{i=0}^{m-1} \overline{a\left[\alpha_{m-i}(t)\right]}-\prod_{i=1}^{m} \overline{b\left[\alpha_{m-i}(t)\right]}= \\
& =\overline{a_{m}(t)-b_{m}(t)}=\overline{\sigma_{A}(t)} .
\end{aligned}
$$

Thus, $\sigma_{A^{*}}(t) \neq 0$ for $t \in \widetilde{\Gamma}_{1}$ if and only if $\sigma_{A}(t) \neq 0$ for $t \in \Gamma_{1}$.

By Corollary 5.2, the set $\widetilde{\Gamma}_{2}=\Gamma_{2}$ is $\alpha_{m-1}$-invariant. Consequently,

$$
\sigma_{A^{*}}(t)=\prod_{i=0}^{m-1} \overline{a\left[\alpha_{-i}(t)\right]} \neq 0, \quad t \in \widetilde{\Gamma}_{2},
$$

if and only if $\sigma_{A}(t)=a_{m}(t) \neq 0$ for $t \in \Gamma_{2}$.

Taking into account that $\alpha$ (and $\alpha_{-1}$ ) is the diffeomorphism, we see that

$$
\sigma_{A^{*}}(t)=\prod_{i=0}^{m-1}\left(\overline{b\left[\alpha_{-i-1}(t)\right]} \cdot\left|\alpha_{-1}^{\prime}\left[\alpha_{-i}(t)\right]\right|\right) \neq 0, \quad t \in \widetilde{\Gamma}_{3},
$$

if and only if

$$
\prod_{i=1}^{m} b\left[\alpha_{-i}(t)\right] \neq 0, \quad t \in \widetilde{\Gamma}_{3} .
$$


Due to Corollary 5.2, the set $\widetilde{\Gamma}_{3}=\Gamma_{3}$ is $\alpha_{m}$-invariant. Thus, (5.16) is equivalent to $\sigma_{A}(t)=$ $b_{m}(t) \neq 0$ for all $t \in \Gamma_{3}$.

Moreover, the condition $\sigma_{A^{*}}(t) \neq 0\left(\sigma_{A}(t) \neq 0\right)$ for all $t \in \Gamma \backslash \widetilde{\Gamma}_{4}$ (respectively, $t \in \Gamma \backslash \Gamma_{5}$ ) implies $\Gamma=\widetilde{\Gamma}_{1} \cup \widetilde{\Gamma}_{2} \cup \widetilde{\Gamma}_{3} \cup \widetilde{\Gamma}_{4}$ (respectively, $\Gamma=\Gamma_{1} \cup \Gamma_{2} \cup \Gamma_{3} \cup \Gamma_{5}$ ). In view of $(5.15)$

$$
\widetilde{\Gamma}_{1} \cup \widetilde{\Gamma}_{2} \cup \widetilde{\Gamma}_{3}=\Gamma \backslash \widetilde{\Gamma}_{4}=\Gamma \backslash \Gamma_{5}=\Gamma_{1} \cup \Gamma_{2} \cup \Gamma_{3} .
$$

Thus, $\sigma_{A}(t) \neq 0$ for all $t \in \Gamma \backslash \Gamma_{5}$ if and only if $\sigma_{A^{*}}(t) \neq 0$ for all $t \in \Gamma \backslash \widetilde{\Gamma}_{4}$. The statement (b) is proved by analogy.

\subsection{Reduction to the case of fixed points}

In this subsection we reduce the investigation of the right invertibility of the operator $A$ with the shift $\alpha$ preserving orientation on $\Gamma$ and having periodic points of multiplicity $m>1$, or changing orientation on $\Gamma$ (then $m=2$ ), to the investigation of the right invertibility of some operator of the same form with the shift $\alpha_{m}$ which has only fixed points.

Theorem 5.7. The operator $A=a I-b W$ is right invertible in $X(\Gamma)$ if and only if the operator $A_{m}:=a_{m} I-b_{m}\left(\alpha_{m-1}\right) W^{m}$ is right invertible in $X(\Gamma)$, and

$$
\left|a_{i}(t)\right|+\left|b\left[\alpha_{i-1}(t)\right]\right|>0 \quad \text { for all } \quad t \in \Gamma_{4} \quad \text { and all } \quad i=1,2, \ldots, m-1 .
$$

Proof. If $m=1$, then the statement is trivial. So, assume that $m>1$.

If the shift $\alpha$ preserves the orientation on $\Gamma$, then fix some $\tau \in \Lambda$ and choose an arbitrary open arc $l$ from the set

$$
\left\{(\tau, \alpha(\tau)),\left(\alpha(\tau), \alpha_{2}(\tau)\right), \ldots,\left(\alpha_{m-1}(\tau), \alpha_{m}(\tau)\right)\right\}
$$

If the shift $\alpha$ changes the orientation on $\Gamma$, then $\alpha$ has exactly two fixed points on $\Gamma$, say $z_{1}$ and $z_{2}$. In this case take $m=2$, and let $l$ be an arbitrary open arc from the set $\left\{\left(z_{1}, z_{2}\right),\left(z_{2}, z_{1}\right)\right\}$. Then $\Gamma \backslash\left\{z_{1}, z_{2}\right\}=l \cup \alpha(l)$.

Let $[X(l)]^{m}$ be the space of all vectors with $m$ components from $X(l)$ and $\sigma_{l}$ be the isomorphism of $X(\Gamma)$ onto $[X(l)]^{m}$ defined by the rule

$$
\left(\sigma_{l} \varphi\right)(t):=\left\{\varphi\left[\alpha_{k}(t)\right]\right\}_{k=0}^{m-1}, \quad t \in l
$$

For $1 \leq i \leq m-1$, define the operators $F_{i}:[X(\Gamma)]^{m-i+1} \rightarrow[X(\Gamma)]^{m-i+1}$ by

$$
F_{i}:=\left[\begin{array}{ccccc}
a_{i} I & -b\left(\alpha_{i-1}\right) I & \ddots & O & O \\
O & a\left(\alpha_{i}\right) I & \ddots & O & O \\
\ddots & \ddots & \ddots & \ddots & \ddots \\
O & O & \ddots & a\left(\alpha_{m-2}\right) I & -b\left(\alpha_{m-2}\right) I \\
-b_{i}\left(\alpha_{m-1}\right) W^{m} & O & \ddots & O & a\left(\alpha_{m-1}\right) I
\end{array}\right] .
$$

Since

$$
\sigma_{l} A \sigma_{l}^{-1} \varphi=F_{1} \varphi \quad \text { for all } \varphi \in[X(l)]^{m}
$$


and since $l$ is an arbitrary connected component in $\Gamma \backslash\left\{\tau, \alpha(\tau), \ldots, \alpha_{m-1}(\tau)\right\}$ or $\Gamma \backslash\left\{z_{1}, z_{2}\right\}$, the operator $A$ is right invertible in $X(\Gamma)$ if and only if the operator $F_{1}$ is right invertible in each space $\left[X\left(\alpha_{k}(l)\right)\right]^{m}, k \in\{0,1, \ldots, m-1\}$. In view of the equality

$$
[X(\Gamma)]^{m}=[X(l)]^{m} \oplus[X(\alpha(l))]^{m} \oplus \ldots \oplus\left[X\left(\alpha_{m-1}(l)\right)\right]^{m},
$$

the operator $A$ is right invertible in the space $X(\Gamma)$ if and only if the operator $F_{1}$ is right invertible in the space $[X(\Gamma)]^{m}$. If the operator $F_{i}, i \in\{1,2, \ldots, m-1\}$, is right invertible in the space $[X(\Gamma)]^{m-i+1}$, then we get the $i$-th relation in

$$
\min _{t \in \Gamma}\left(\left|a_{i}(t)\right|+\left|b\left[\alpha_{i-1}(t)\right]\right|\right)>0, \quad i=1,2, \ldots, m-1 .
$$

Indeed, otherwise all the elements of the $i$-th row have zero at a point $t \in \Gamma$, which implies that $\operatorname{Im} F_{i} \neq[X(\Gamma)]^{m-i+1}$.

If (5.18) holds for some $i \in\{1,2, \ldots, m-1\}$, then there is a function $g^{(i)} \in C(\Gamma)$ such that the function $f^{(i)}:=a_{i} g^{(i)}+b\left(\alpha_{i-1}\right)$ is bounded away from zero. Consequently, the operator

$$
C_{i}:=\left[\begin{array}{cc}
a_{i} I & -b\left(\alpha_{i-1}\right) I \\
\left(1 / f^{(i)}\right) I & \left(g^{(i)} / f^{(i)}\right) I
\end{array}\right], \quad i=1,2, \ldots, m-1,
$$

is invertible in the space $[X(\Gamma)]^{2}$, and the following equality holds:

$$
F_{i}= \begin{cases}{\left[\begin{array}{cc}
I & O_{1 \times(m-i)} \\
D_{i} & F_{i+1}
\end{array}\right]\left[\begin{array}{cc}
C_{i} & O_{2 \times(m-i-1)} \\
O_{(m-i-1) \times 2} & I_{m-i-1}
\end{array}\right],} & i=1,2, \ldots, m-2, \\
{\left[\begin{array}{cc}
I & O \\
D_{i} & F_{i+1}
\end{array}\right] C_{i},} & i=m-1,\end{cases}
$$

where the operator $D_{i}: X(\Gamma) \rightarrow[X(\Gamma)]^{m-i}$ is defined by the formula

$$
D_{i}:= \begin{cases}\operatorname{column}[-\frac{a\left(\alpha_{i}\right)}{f^{(i)}} I, \overbrace{O, \ldots, O}^{m-2-i},-b_{i}\left(\alpha_{m-1}\right) W^{m} \frac{g^{(i)}}{f^{(i)}} I], & i=1,2, \ldots, m-2, \\ -\frac{a\left(\alpha_{m-1}\right)}{f^{(m-1)}} I-b_{m-1}\left(\alpha_{m-1}\right) W^{m} \frac{g^{(m-1)}}{f^{(m-1)}} I, & i=m-1,\end{cases}
$$

$I_{k}$ is the identity operator in the space $[X(\Gamma)]^{k}, O_{k \times p}$ is the zero operator from the space $[X(\Gamma)]^{p}$ into the space $[X(\Gamma)]^{k}$, and $F_{m}:=A_{m}$.

Since the second term on the right of (5.19) is invertible in the space $[X(\Gamma)]^{m-i+1}$, the right invertibility of the operator $F_{i}$ in the space $[X(\Gamma)]^{m-i+1}$ is equivalent to the right invertibility of the first term on the right of (5.19) in the space $[X(\Gamma)]^{m-i+1}$, which in its turn is equivalent to the right invertibility of the operator $F_{i+1}$ in the space $[X(\Gamma)]^{m-i}$.

then

Indeed, if $F_{i+1}$ is right invertible in $[X(\Gamma)]^{m-i}$ and $F_{i+1}^{(-1)}$ is one of its right inverses,

$$
\left[\begin{array}{cc}
I & O_{1 \times(m-i)} \\
D_{i} & F_{i+1}
\end{array}\right]\left[\begin{array}{cc}
I & O_{1 \times(m-i)} \\
-F_{i+1}^{(-1)} D_{i} & F_{i+1}^{(-1)}
\end{array}\right]=\left[\begin{array}{cc}
I & O_{1 \times(m-i)} \\
O_{(m-i) \times 1} & I_{m-i}
\end{array}\right]
$$


Hence, the first term on the right of (5.19) is right invertible. On the other hand, if

$$
\left[\begin{array}{cc}
I & O_{1 \times(m-i)} \\
D_{i} & F_{i+1}
\end{array}\right]\left[\begin{array}{cc}
B_{1} & B_{2} \\
B_{3} & B_{4}
\end{array}\right]=\left[\begin{array}{cc}
B_{1} & B_{2} \\
D_{i} B_{1}+F_{i+1} B_{3} & D_{i} B_{2}+F_{i+1} B_{4}
\end{array}\right]\left[\begin{array}{cc}
I & O_{1 \times(m-i)} \\
O_{(m-i) \times 1} & I_{m-i}
\end{array}\right]
$$

then $B_{2}=O_{1 \times(m-i)}$. Hence, $F_{i+1} B_{4}=I_{m-i}$, that is, the operator $F_{i+1}$ is right invertible in the space $[X(\Gamma)]^{m-i}$.

Thus, the operator $A$ is right invertible in the space $X(\Gamma)$ if and only if the operator $A_{m}=F_{m}$ is right invertible in the space $X(\Gamma)$ and, for every $i \in\{1,2, \ldots, m-1\}$, inequality (5.18) holds. Clearly, (5.18) implies (5.17), so the necessity is proved.

It remains to prove that $(5.17)$ and the right invertibility of $A_{m}$ imply (5.18). From (5.4)-(5.6), (3.1)-(3.2), (4.10) and Proposition 5.1 it follows that the functions $\eta_{i}^{ \pm}, i \in\{0,1\}$, defined for operators $A_{m}=a_{m} I-b_{m}\left(\alpha_{m-1}\right) W_{\alpha_{m}}$ and $A$, coincide. Hence, for these operators, the corresponding sets $\Gamma_{j}, j \in\{1,2, \ldots, 5\}$, coincide as well.

If the operator $A_{m}$ is right invertible, then, by Theorem 4.6,

$$
\sigma_{A_{m}}(t) \neq 0 \text { for all } t \in \Gamma \backslash \Gamma_{4},
$$

where

$$
\sigma_{A_{m}}(t):=\left\{\begin{array}{cl}
a_{m}(t)-b_{m}\left[\alpha_{m-1}(t)\right], & t \in \Gamma_{1}, \\
a_{m}(t), & t \in \Gamma_{2}, \\
-b_{m}\left[\alpha_{m-1}(t)\right], & t \in \Gamma_{3}, \\
0, & t \in \Gamma \backslash\left(\Gamma_{1} \cup \Gamma_{2} \cup \Gamma_{3}\right) .
\end{array}\right.
$$

It is easy to see that $b_{m}\left[\alpha_{m-1}(t)\right]=b_{m}(t)$ for $t \in \Gamma_{1}$. In view of Corollary 5.2, the set $\Gamma_{3}$ is $\alpha_{m-1}$-invariant. Hence, $b_{m}\left[\alpha_{m-1}(t)\right] \neq 0$ for all $t \in \Gamma_{3}$ if and only if $b_{m}(t) \neq 0$ for all $t \in \Gamma_{3}$. Thus, (5.20) is equivalent to

$$
\sigma_{A}(t) \neq 0 \quad \text { for all } t \in \Gamma \backslash \Gamma_{4}
$$

From (5.21) it follows that

$$
\left|a_{i}(t)\right|+\left|b\left[\alpha_{i-1}(t)\right]\right|>0 \text { for all } \quad t \in \Gamma \backslash \Gamma_{4} \quad \text { and all } i=1,2, \ldots, m-1 .
$$

Indeed, assume the contrary: for some $i \in\{1,2, \ldots, m-1\}$ there is a point $t_{0} \in \Gamma \backslash \Gamma_{4}$ such that $\left|a_{i}\left(t_{0}\right)\right|=\left|b\left[\alpha_{i-1}\left(t_{0}\right)\right]\right|=0$. Hence, $a_{m}\left(t_{0}\right)=0$ and $b_{m}\left(t_{0}\right)=0$. Consequently, $\sigma_{A}\left(t_{0}\right)=0$ and we get the contradiction. Clearly, (5.17) and (5.22) imply (5.18), which completes the proof of the sufficiency.

\subsection{Proof of Theorem 5.3}

The case of $m=1$ was considered in Theorem 4.6. Assume that $m>1$ and consider the case of the right invertiblity of $A$.

In view of Theorem 5.7, the right invertibility of $A$ is equivalent to (5.17) and the right invertibility of $A_{m}$. By Theorem 4.6, the right invertibility of $A_{m}$ is equivalent to (5.20) and the condition

for every $t \in \Gamma_{4}$ there is $k_{0} \in \mathbf{Z}$ such that

$$
a_{m}\left[\alpha_{m k}(t)\right] \neq 0 \text { for } k<k_{0}, \quad b_{m}\left[\alpha_{m k+m-1}(t)\right] \neq 0 \text { for } k \geq k_{0} \text {. }
$$


Let us show that the conjunction of $(5.17)$ and $(5.23)$ is equivalent to the condition

for every $t \in \Gamma_{4}$ there are $s \in\{0,1, \ldots, m-1\}$ and $k_{0} \in \mathbf{Z}$ such that

$$
a\left[\alpha_{k}(t)\right] \neq 0 \text { for } k<m k_{0}+s, \quad b\left[\alpha_{k}(t)\right] \neq 0 \text { for } k \geq m k_{0}+s .
$$

Indeed, condition (5.23) is equivalent to the condition

$$
\begin{gathered}
\text { for every } t \in \Gamma_{4} \text { there is } k_{0} \in \mathbf{Z} \text { such that } \\
a\left[\alpha_{k}(t)\right] \neq 0 \text { for } k<m k_{0}, \quad b\left[\alpha_{k}(t)\right] \neq 0 \text { for } k \geq m k_{0}+m-1 .
\end{gathered}
$$

Since the set $\Gamma_{4}$ is invariant with respect to the shift $\alpha_{m k_{0}}$, (5.17) is equivalent to the condition

$$
\begin{gathered}
\text { for every } t \in \Gamma_{4} \text { and every } i \in\{1,2, \ldots, m-1\}, \\
\prod_{j=0}^{i-1}\left|a\left[\alpha_{m k_{0}+j}(t)\right]\right|+\left|b\left[\alpha_{m k_{0}+i-1}(t)\right]\right|>0 .
\end{gathered}
$$

Fix $t \in \Gamma_{4}$. If $a\left[\alpha_{m k_{0}+i}(t)\right] \neq 0$ for all $i \in\{0,1, \ldots, m-2\}$, then from (5.25) we get (5.24) with $s=m-1$. Otherwise there exists an $s \in\{0,1, \ldots, m-2\}$ such that

$$
a\left[\alpha_{m k_{0}+i}(t)\right] \neq 0, \quad i \in\{0,1, \ldots, s-1\}, \quad a\left[\alpha_{m k_{0}+s}(t)\right]=0 .
$$

Then $\prod_{j=0}^{i-1} a\left[\alpha_{m k_{0}+j}(t)\right]=0$ for $i \in\{s+1, \ldots, m-1\}$. In that case (5.26) implies

$$
b\left[\alpha_{m k_{0}+i-1}(t)\right] \neq 0, \quad i \in\{s+1, \ldots, m-1\} .
$$

From (5.27), (5.28) and (5.25) we get (5.24) with $s \in\{0,1, \ldots, m-2\}$.

On the other hand, if (5.24) holds, then the conditions (5.25) and (5.26) are fulfilled. But as it was said above, (5.26) is equivalent to (5.17), and (5.25) is equivalent to (5.23). So, we get that we need. It remains to note that condition (5.24) is equivalent to $R\left(\Gamma_{4}\right)$.

Thus, we have proved that the right invertibility of $A$ is equivalent to $(5.20)$ and the property $R\left(\Gamma_{4}\right)$. But, as was shown in the proof of Theorem 5.7, the properties (5.20) and (5.17) are equivalent. The case of the right invertibility is considered.

The case of the left invertibility is reduced to the previous one by passing to adjoint operators and applying Proposition 5.5(a) and Lemma 5.6(a).

Corollary 5.8. The operator $A$ is invertible in $X(\Gamma)$ if and only if $\sigma_{A}(t) \neq 0$ for all $t \in \Gamma$.

Proof. The condition $\sigma_{A}(t) \neq 0$ for all $t \in \Gamma \backslash \Gamma_{4}$ (respectively, $\left.t \in \Gamma \backslash \Gamma_{5}\right)$ implies that $\Gamma_{5}=\emptyset$ (respectively, $\Gamma_{4}=\emptyset$ ). Thus, in the case of the invertibility of $A$, from Theorem 5.3 we get $\Gamma_{4}=\Gamma_{5}=\emptyset$, and conditions (5.9), (5.8) degenerate to $\sigma_{A}(t) \neq 0$ for all $t \in \Gamma=\Gamma_{1} \cup \Gamma_{2} \cup \Gamma_{3}$.

On the other hand, the condition $\sigma_{A}(t) \neq 0$ for all $t \in \Gamma$ implies that $\Gamma=\Gamma_{1} \cup \Gamma_{2} \cup \Gamma_{3}$, that is, $\Gamma_{4}=\Gamma_{5}=\emptyset$. Hence, by Theorem 5.3, the operator $A$ is simultaneously right and left invertible.

\subsection{Spectrum of the weighted shift operator}

The spectrum of the weighted shift operator is calculated in [13, Lemma 6.10] in the case of only two fixed points. Now we generalize this result to the case of arbitrary nonempty set of periodic points. 
For a continuous function $d$ and a number $m \in \mathbf{N}$, consider the two functions

$$
\begin{aligned}
\delta(t) & :=\left|d_{m}(t)\right| \min \left\{\left|\alpha_{m}^{\prime}(t)\right|^{-\alpha_{X}},\left|\alpha_{m}^{\prime}(t)\right|^{-\beta_{X}}\right\}, \\
\Delta(t) & :=\left|d_{m}(t)\right| \max \left\{\left|\alpha_{m}^{\prime}(t)\right|^{-\alpha_{X}},\left|\alpha_{m}^{\prime}(t)\right|^{-\beta_{X}}\right\} .
\end{aligned}
$$

Theorem 5.9. The spectrum of the weighted shift operator $d W$ with a weight (coefficient) $d \in C(\Gamma)$ in a reflexive $r .-i$. space $X(\Gamma)$ of fundamental type with nontrivial Boyd indices $\alpha_{X}, \beta_{X}$ has the form

$$
\sigma(d W)=\left(\bigcup_{t \in \Gamma \backslash \Phi}\left\{z \in \mathbf{C}: z^{m}=d_{m}(t)\right\}\right) \cup\left(\bigcup_{\gamma \subset \Phi \backslash \Lambda} \Omega(d, \gamma)\right) \cup\left(\bigcup_{\tau \in Y^{\prime}} \Psi(d, \tau)\right)
$$

where $\gamma$ are connected components of $\Phi \backslash \Lambda$,

$$
\begin{gathered}
\Omega(d, \gamma)= \begin{cases}\left\{z \in \mathbf{C}: \min _{\tau \in \Lambda \cap \bar{\gamma}} \delta(\tau) \leq|z|^{m} \leq \max _{\tau \in \Lambda \cap \bar{\gamma}} \Delta(\tau)\right\} & \text { if } d_{m} \in G C(\bar{\gamma}), \\
\left\{z \in \mathbf{C}:|z|^{m} \leq \max _{\tau \in \Lambda \cap \bar{\gamma}} \Delta(\tau)\right\} & \text { if } d_{m} \notin G C(\bar{\gamma}),\end{cases} \\
\Psi(d, \tau)=\left\{z \in \mathbf{C}: \delta(\tau) \leq|z|^{m} \leq \Delta(\tau)\right\} .
\end{gathered}
$$

Proof. Corollary 5.8 and the equivalence of (5.21) and (5.20) imply that the operator $B:=z I-d W, z \in \mathbf{C}$, is not invertible in $X(\Gamma)$ if and only if the operator $B_{m}=z^{m} I-d_{m} W_{\alpha_{m}}$ is not invertible in $X(\Gamma)$, where $m$ is the multiplicity of periodic points of the shift $\alpha$ if $\alpha$ preserves the orientation on $\Gamma$, and $m=2$ otherwise. Clearly, the shift $\alpha_{m}$ has only fixed points, and we can apply Theorem 4.4 to the operator $B_{m}$. It is easy to see that

$$
\eta_{0}(t)=|z|^{m}-\delta(t), \quad \eta_{1}(t)=|z|^{m}-\Delta(t) .
$$

Hence, by Theorem 4.4, the spectrum of the operator $d W$ has the form

$$
\begin{aligned}
& \sigma(d W)=\left(\bigcup_{\gamma \subset \Phi \backslash \Lambda}\left\{z \in \mathbf{C}: z^{m} I-d_{m} W_{\alpha_{m}} \text { is not invertible in } X(\gamma)\right\}\right) \\
& \cup\left(\bigcup_{t \in \Gamma \backslash \Phi}\left\{z \in \mathbf{C}: z^{m}=d_{m}(t)\right\}\right) \cup\left(\bigcup_{\tau \in Y^{\prime}}\left\{z \in \mathbf{C}:\left(|z|^{m}-\delta(\tau)\right)\left(|z|^{m}-\Delta(\tau)\right) \leq 0\right\}\right) .
\end{aligned}
$$

By [13, Lemma 6.10] and Lemma 2.3, for $\gamma \in \Phi \backslash \Lambda$,

$$
\left\{z \in \mathbf{C}: z^{m} I-d_{m} W_{\alpha_{m}} \text { is not invertible in } X(\gamma)\right\}=\Omega(d, \gamma) .
$$

Since $0 \leq \delta(t) \leq \Delta(t)$, the inequality $\left(|z|^{m}-\delta(t)\right)\left(|z|^{m}-\Delta(t)\right) \leq 0$ is equivalent to the inequality $\delta(t) \leq|z|^{m} \leq \Delta(t)$. Hence, for $\tau \in Y^{\prime}$,

$$
\left\{z \in \mathbf{C}:\left(|z|^{m}-\delta(\tau)\right)\left(|z|^{m}-\Delta(\tau)\right) \leq 0\right\}=\Psi(d, \tau)
$$


In particular, if $\gamma=\left(\tau_{-}, \tau_{+}\right)$is an arc, the endpoints of which are fixed points of the shift $\alpha$ and $\Lambda \cap \gamma=\emptyset$, then from the description of the set $\Omega(1, \gamma)$ we see that the spectral radius of the shift operator $W$ in $X(\gamma)$ is given by (see also [13], Lemma 6.10])

$$
\begin{aligned}
r(W ; X(\gamma)) & =\max _{\tau \in\left\{\tau_{-}, \tau_{+}\right\}} \max \left\{\left|\alpha^{\prime}(\tau)\right|^{-\alpha_{X}},\left|\alpha^{\prime}(\tau)\right|^{-\beta_{X}}\right\} \\
& =\max \left\{r\left(W ; L^{1 / \alpha_{X}}(\gamma)\right), r\left(W ; L^{1 / \beta_{X}}(\gamma)\right)\right\}
\end{aligned}
$$

This simple example shows, that estimate (2.12) in Theorem 2.4 is sharp.

Note that Theorem 5.9 were obtained for the case of reflexive Orlicz spaces by V. Aslanov and the second author [6] (see also [5], [14, Corollary 2.1]).

Remark. We do not use the restriction on r.-i. spaces to be of fundamental type in the proofs of all results of this paper, except for Theorem 4.1. But the proof of Theorem 4.1 (see [13]) essentially depends on this restriction.

Problem. Is it possible to drop the restrictions $\alpha_{X}=p_{X}$ and $\beta_{X}=q_{X}$ ?

\section{References}

[1] Abramovich, Yu., Arenson, E., and Kitover, A.: Banach $C(K)$-modules and operators preserving disjointness. Pitman Research Notes in Mathematics Series 277, Longman Scientific \& Technical, New York 1992.

[2] Antonevich, A. B.: Linear Functional Equations. Operator Approach. University Press, Minsk 1988 (Russian). English transl.: Birkhäuser Verlag, Basel, Boston, Berlin 1995.

[3] Antonevich, A., and Lebedev, A.: Functional-Differential Equations: I. $C^{*}$-theory. Longman Scientific \& Technical, Harlow 1994.

[4] Antonevich, A., Belousov, M., and Lebedev, A.: Functional Differential Equations: II. C*-applications. Parts 1-2. Longman, Harlow 1998.

[5] Aslanov, V.: Functional and Singular Integral Operators with a Shift in Orlicz Spaces. Ph. D. Thesis, Baku, 1992 (Russian).

[6] Aslanov, V., and Karlovich, Yu. I.: One-sided invertibility of functional operators in reflexive Orlicz spaces. Dokl. Akad. Nauk AzSSR 45 (1989), no. 11-12, 3-7 (Russian).

[7] Bennett, C., and Sharpley, R.: Interpolation of Operators. Academic Press, Boston 1988.

[8] Boyd, D. W.: Indices of function spaces and their relationship to interpolation. Canad. J. Math. 21 (1969), 1245-1254.

[9] Chicone, C., and Latushkin, Yu.: Evolution Semigroups in Dynamical Systems and Differential Equations. American Mathematical Society, Providence, RI, 1999.

[10] FeHÉr, F.: Indices of Banach function spaces and spaces of fundamental type. J. Approx. Theory 37 (1983), 12-28. 
[11] Gohberg, I., and Krupnik, N.: One-Dimensional Linear Singular Integral Equations. Vol. 1. Birkhäuser Verlag, Basel, Boston, Berlin 1992 (Russian original: Shtiintsa, Kishinev 1973).

[12] Kantorovich, L. V., and Akilov, G. P.: Functional analysis. Nauka, Moscow, 3rd ed., 1984 (in Russian). English transl.: Pergamon Press, Oxford, 2nd ed., 1982.

[13] Karlovich, A. Yu.: Criteria for one-sided invertibility of a functional operator in rearrangement-invariant spaces of fundamental type, to appear in Mathematische Nachrichten.

[14] Karlovich, Yu. I.: The continuous invertibility of functional operators in Banach spaces. Dissertationes Math. (Rozprawy Mat.) 340 (1995), 115-136.

[15] Karlovich, Yu. I., and Kravchenko, V. G.: A Noether theory for a singular integral operator with a shift having periodic points. Soviet Math. Dokl. 17 (1976), $1547-1551$.

[16] Kravchenko, V. G., and Litvinchuk, G. S.: Introduction to the Theory of Singular Integral Operators with Shift. Series: Mathematics and its applications 289, Kluwer Academic Publishers, Dordrecht, Boston, London 1994.

[17] Krein, S. G., Petunin, Ju. I., and Semenov, E. M.: Interpolation of Linear Operators. Nauka, Moscow 1978 (Russian). English transl.: AMS Translations of Mathematical Monographs 54, Providence, R.I., 1982.

[18] Kurbatov, V. G.: Functional Differential Operators and Equations. Series: Mathematics and its applications 473, Kluwer Academic Publishers, Dordrecht, Boston, London 1999.

[19] Lindenstrauss, J. and Tzafriri, L.: Classical Banach Spaces. Function Spaces. Springer Verlag, New York, Berlin 1979.

[20] Litvinchuk, G. S.: Boundary Value Problems and Singular Integral Equations with Shift. Nauka, Moscow 1977 (Russian).

[21] Maligranda, L.: Indices and interpolation, Dissertationes Math. (Rozprawy Mat.) 234 (1985), 1-49.

[22] Maligranda, L.: Orlicz Spaces and Interpolation. Sem. Math. 5, Dep. Mat., Univ. Estadual de Campinas, Campinas SP, Brazil, 1989.

[23] Mardiev, R.: A criterion for the semi-Noetherian property of one class of singular integral operators with a non-Carleman shift. Dokl. Akad Nauk UzSSR (1985), no. 2, 5-7 (Russian).

[24] Mardiev, R.: A criterion for $n(d)$-normality of singular integral operators with a shift having periodic points in Lebesgue spaces. Samarkand, 1988, 41 pp. Manuscript no. 821-Uz88, deposited at UzNIINTI (Russian).

[25] ZipPIN, M.: Interpolation of operators of weak type between rearrangement invariant spaces. J. Functional Analysis 7 (1971), 267-284. 
A. Yu. Karlovich

Departamento de Matemática

Instituto Superior Técnico

Av. Rovisco Pais

1049-001, Lisboa,

Portugal

E-mail: akarlov@math.ist.utl.pt http://www.math.ist.utl.pt/akarlov

MSC 1991: Primary 39B32, 47B38

Secondary 46E30, 47A10
Yu. I. Karlovich

CINVESTAV del I.P.N.

Departamento de Matemáticas

Apartado Postal 14-740

07000, Mexico, D.F.,

Mexico

E-mail: karlovic@math.cinvestav.mx 